\title{
MAGIC GAMMA-RAY TELESCOPE OBSERVATION OF THE PERSEUS CLUSTER OF GALAXIES: IMPLICATIONS FOR COSMIC RAYS, DARK MATTER, AND NGC 1275
}

J. Aleksić ${ }^{1}$, L. A. Antonelli ${ }^{2}$, P. Antoranz ${ }^{3}$, M. Backes ${ }^{4}$, C. Baixeras ${ }^{5}$, S. Balestra ${ }^{3}$, J. A. Barrio ${ }^{3}$, D. Bastieri ${ }^{6}$, J. Becerra González ${ }^{7}$, W. BednareK ${ }^{8}$, A. Berdyugin ${ }^{9}$, K. Berger $^{9}$, E. Bernardini ${ }^{10}$, A. Biland ${ }^{11}$, R. K. Bock ${ }^{6,12}$, G. BonNoli ${ }^{2}$, P. Bordas ${ }^{13}$, D. Borla Tridon ${ }^{12}$, V. Bosch-RAMON ${ }^{13}$, D. Bose ${ }^{3}$, I. Braun ${ }^{11}$, T. Bretz ${ }^{14}$, D. BritzGer ${ }^{12}$, M. Camara ${ }^{3}$, E. Carmona ${ }^{12}$, A. Carosi ${ }^{2}$, P. Colin ${ }^{12}$, S. Commichau ${ }^{11}$, J. L. Contreras ${ }^{3}$, J. Cortina ${ }^{1}$, M. T. Costado ${ }^{7,15}$, S. Covino ${ }^{2}$, F. Dazzi ${ }^{16,31}$, A. De Angelis ${ }^{16}$, E. De Cea Del Pozo ${ }^{17}$, R. De los Reyes ${ }^{3,32}$, B. De Lotto ${ }^{16}$, M. De Maria ${ }^{16}$, F. De Sabata ${ }^{16}$, C. Delgado Mendez ${ }^{7,33}$, M. Doert ${ }^{4}$, A. Domínguez ${ }^{18}$, D. Dominis Prester ${ }^{19}$, D. Dorner ${ }^{11}$, M. Doro ${ }^{6}$, D. Elsaesser ${ }^{14}$, M. Errando ${ }^{1}$, D. Ferenc ${ }^{19}$, M. V. Fonseca ${ }^{3}$, L. Font ${ }^{5}$, N. Galante ${ }^{12}$, R. J. García López ${ }^{7,15}$,

M. GarczarczyK ${ }^{7}$, M. Gaug ${ }^{7}$, N. Godinovic ${ }^{19}$, D. Hadasch ${ }^{17}$, A. Herrero ${ }^{7}, 15$, D. Hildebrand ${ }^{11}$, D. HöHNe-MönCh ${ }^{14}$,

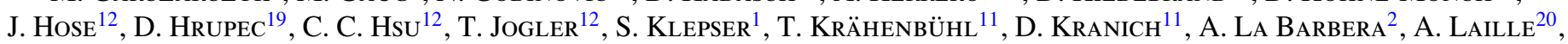

E. LEONARDO ${ }^{21}$, E. LindFORS ${ }^{9}$, S. LOMBARDI ${ }^{6}$, F. LONGO ${ }^{16}$, M. LÓPEZ $Z^{6}$, E. LORENZ ${ }^{11,12}$, P. MAJUMdAR ${ }^{10}$, G. MANEVA ${ }^{22}$, N. Mankuzhiyil ${ }^{16}$, K. Mannheim ${ }^{14}$, L. Maraschi ${ }^{2}$, M. Mariotti ${ }^{6}$, M. Martínez ${ }^{1}$, D. Mazin ${ }^{1}$, M. Meucci ${ }^{21}$, J. M. Miranda ${ }^{3}$, R. Mirzoyan ${ }^{12}$, H. Miyamoto ${ }^{12}$, J. Moldón ${ }^{13}$, M. Moles ${ }^{18}$, A. Moralejo ${ }^{1}$, D. Nieto ${ }^{3}$, K. Nilsson ${ }^{9}$, J. Ninkovic $^{12}$, R. ORito $^{12}$, I. Oya ${ }^{3}$, S. Paiano ${ }^{6}$, R. Paoletti ${ }^{21}$, J. M. Paredes ${ }^{13}$, S. Partini ${ }^{21}$, M. Pasanen ${ }^{9}$, D. Pascoli ${ }^{6}$, F. Pauss ${ }^{11}$, R. G. Pegna ${ }^{21}$, M. A. Perez-Torres ${ }^{18}$, M. Persic ${ }^{16,23}$, L. Peruzzo ${ }^{6}$, F. Prada ${ }^{18}$, E. Prandini ${ }^{6}$, N. Puchades ${ }^{1}$, I. Puljak ${ }^{19}$, I. Reichardt ${ }^{1}$,

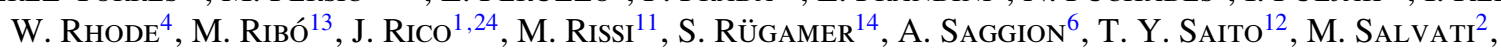

M. A. Sánchez-Conde ${ }^{18}$, K. Satalecka ${ }^{10}$, V. Scalzotto ${ }^{6}$, V. Scapin ${ }^{16}$, C. Schultz ${ }^{6}$, T. Schweizer ${ }^{12}$, M. ShaYduk ${ }^{12}$, S. N. Shore ${ }^{25}$, A. Sierpowska-Bartosik ${ }^{8}$, A. Sillanpä̈̈ ${ }^{9}$, J. SitareK ${ }^{8,12}$, D. SobcZYNSKa ${ }^{8}$, F. Spanier ${ }^{14}$, S. Spiro ${ }^{2}$, A. Stamerra ${ }^{21}$, B. Steinke ${ }^{12}$, J. C. Struebig ${ }^{14}$, T. Suric ${ }^{19}$, L. Takalo ${ }^{9}$, F. Tavecchio $^{2}$, P. Temnikov $^{22}$, T. Terzic ${ }^{19}$,

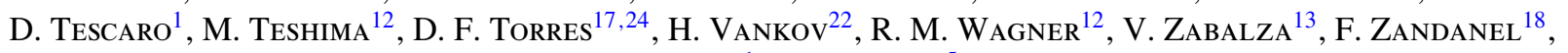
R. ZANIN ${ }^{1}$, J. ZAPATERO

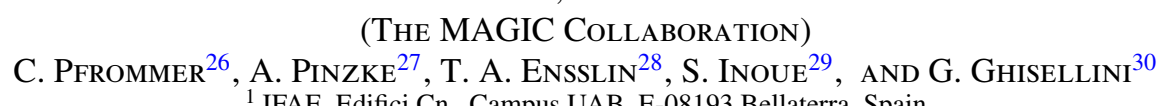 \\ ${ }^{1}$ IFAE, Edifici Cn., Campus UAB, E-08193 Bellaterra, Spain \\ ${ }^{2}$ INAF National Institute for Astrophysics, I-00136 Rome, Italy \\ ${ }^{3}$ Universidad Complutense, E-28040 Madrid, Spain \\ ${ }^{4}$ Technische Universität Dortmund, D-44221 Dortmund, Germany \\ ${ }^{5}$ Universitat Autònoma de Barcelona, E-08193 Bellaterra, Spain \\ ${ }^{6}$ Università di Padova and INFN, I-35131 Padova, Italy \\ ${ }^{7}$ Instituto de Astrofísica de Canarias, E-38200 La Laguna, Tenerife, Spain \\ ${ }^{8}$ University of Łódź, PL-90236 Lodz, Poland \\ ${ }^{9}$ Tuorla Observatory, University of Turku, FI-21500 Piikkiö, Finland \\ ${ }^{10}$ Deutsches Elektronen-Synchrotron (DESY), D-15738 Zeuthen, Germany \\ ${ }^{11}$ ETH Zurich, CH-8093 Zurich, Switzerland \\ ${ }^{12}$ Max-Planck-Institut für Physik, D-80805 München, Germany \\ ${ }^{13}$ Universitat de Barcelona (ICC/IEEC), E-08028 Barcelona, Spain \\ ${ }^{14}$ Universität Würzburg, D-97074 Würzburg, Germany \\ ${ }^{15}$ Departmento de Astrofisica, Universidad, E-38206 La Laguna, Tenerife, Spain \\ ${ }^{16}$ Università di Udine, and INFN Trieste, I-33100 Udine, Italy \\ ${ }^{17}$ Institut de Ciències de l'Espai (IEEC-CSIC), E-08193 Bellaterra, Spain \\ ${ }^{18}$ Instituto de Astrofísica de Andalucía (CSIC), E-18080 Granada, Spain; fabio@iaa.es \\ ${ }^{19}$ Croatian MAGIC Consortium, Institute R. Boskovic, University of Rijeka and University of Split, HR-10000 Zagreb, Croatia \\ ${ }^{20}$ University of California, Davis, CA 95616-8677, USA \\ ${ }^{21}$ Università di Siena, and INFN Pisa, I-53100 Siena, Italy \\ ${ }^{22}$ Institute for Nuclear Research and Nuclear Energy, BG-1784 Sofia, Bulgaria \\ ${ }^{23}$ INAF/Osservatorio Astronomico and INFN, I-34143 Trieste, Italy \\ ${ }^{24}$ ICREA, E-08010 Barcelona, Spain \\ ${ }^{25}$ Università di Pisa, and INFN Pisa, I-56126 Pisa, Italy \\ ${ }^{26}$ CITA, University of Toronto, M5S 3H8 Toronto, Canada; pfrommer@ cita.utoronto.ca \\ ${ }^{27}$ Stockholm University, SE-106 91 Stockholm, Sweden \\ ${ }^{28}$ Max-Planck-Institut für Astrophysik, D-85740 Garching, Germany \\ ${ }^{29}$ Kyoto University, 606-8502 Kyoto, Japan \\ ${ }^{30}$ INAF National Institute for Astrophysics, I-23807 Merate, Italy \\ Received 2009 September 17; accepted 2009 December 30; published 2010 January 20
}

\begin{abstract}
The Perseus galaxy cluster was observed by the MAGIC Cherenkov telescope for a total effective time of $24.4 \mathrm{hr}$ during 2008 November and December. The resulting upper limits on the $\gamma$-ray emission above $100 \mathrm{GeV}$ are in the range of $4.6-7.5 \times 10^{-12} \mathrm{~cm}^{-2} \mathrm{~s}^{-1}$ for spectral indices from -1.5 to -2.5 , thereby constraining the emission produced by cosmic rays, dark matter annihilations, and the central radio galaxy NGC 1275. Results are compatible with cosmological cluster simulations for the cosmic-ray-induced $\gamma$-ray emission, constraining the average cosmic ray-to-thermal pressure to $<4 \%$ for the cluster core region ( $<8 \%$ for the entire cluster).
\end{abstract}


Using simplified assumptions adopted in earlier work (a power-law spectrum with an index of -2.1 , constant cosmic ray-to-thermal pressure for the peripheral cluster regions while accounting for the adiabatic contraction during the cooling flow formation), we would limit the ratio of cosmic ray-to-thermal energy to $E_{\mathrm{CR}} / E_{\mathrm{th}}<3 \%$. Improving the sensitivity of this observation by a factor of about 7 will enable us to scrutinize the hadronic model for the Perseus radio mini-halo: a non-detection of $\gamma$-ray emission at this level implies cosmic ray fluxes that are too small to produce enough electrons through hadronic interactions with the ambient gas protons to explain the observed synchrotron emission. The upper limit also translates into a level of $\gamma$-ray emission from possible annihilations of the cluster dark matter (the dominant mass component) that is consistent with boost factors of $\sim 10^{4}$ for the typically expected dark matter annihilation-induced emission. Finally, the upper limits obtained for the $\gamma$-ray emission of the central radio galaxy NGC 1275 are consistent with the recent detection by the Fermi-LAT satellite. Due to the extremely large Doppler factors required for the jet, a one-zone synchrotron self-Compton model is implausible in this case. We reproduce the observed spectral energy density by using the structured jet (spine-layer) model which has previously been adopted to explain the high-energy emission of radio galaxies.

Key words: galaxies: clusters: individual (Perseus) - gamma rays: general

\section{INTRODUCTION}

Clusters of galaxies provide us with the opportunity to study an "ecosystem," a volume that is a high-density microcosm of the rest of the Universe. Clusters of galaxies are the largest and most massive gravitationally bound systems in the Universe, with radii of a few $\mathrm{Mpc}$ and total masses $M \sim\left(10^{14}-0^{15}\right)$ $M_{\odot}$, of which galaxies, gas, and dark matter (DM) contribute roughly $5 \%, 15 \%$, and $80 \%$, respectively (see, e.g., Sarazin 1988; Kochanek et al. 2003; Voit 2005 for a general overview). While no cluster has been firmly detected as a $\gamma$-ray source so far (Reimer et al. 2003; Perkins et al. 2006; Perkins 2008; Aharonian et al. 2009a, 2009b; Domainko et al. 2009; Galante et al. 2009; Kiuchi et al. 2009; Acciari et al. 2009), they are expected to be significant $\gamma$-ray emitters on the following general grounds: (1) Clusters are actively evolving objects and being assembled today, in the latest and most energetic phase of hierarchical structure formation. (2) Clusters serve as cosmic energy reservoirs for powerful sources such as radio galaxies and supernova-driven galactic winds. (3) Finally, clusters contain large amounts of gas with embedded magnetic fields, often showing direct evidence for shocks and turbulence as well as relativistic particles. For recent reviews regarding non-thermal processes in clusters as well as numerical simulations, see Blasi et al. (2007) and Dolag et al. (2008).

In the cosmological hierarchic clustering model, large-scale structures grow hierarchically through merging and accretion of smaller systems into larger ones, and clusters are the latest and most massive objects to form (e.g., Peebles 1993). Recently, high resolution X-ray observations by the Chandra and XMMNewton orbiting telescopes provided confirmation of this picture (e.g., Rosati et al. 2002; Voit 2005). During the course of cluster assembly, energies of the order of the final gas binding energy $E_{b} \sim 3 \times\left(10^{61}-10^{63}\right)$ erg should be dissipated through merger and accretion shocks (collectively called "structure formation shocks") as well as turbulence. The energy is expected to be dissipated on a dynamical time scale of $\tau_{\text {dyn }} \sim 1$ Gyr. Hence the corresponding rates of energy release are $L \sim$ $\left(10^{45}-10^{47}\right)$ erg $\mathrm{s}^{-1}$, so even a small fraction of this energy channeled into non-thermal particles can be of major observable consequence. Shocks and turbulence are also likely to accelerate non-thermal electrons and protons to high energies (e.g., Jaffe 1977; Schlickeiser et al. 1987; Brunetti et al. 2001, 2004, 2007;

\footnotetext{
${ }^{31}$ Supported by INFN Padova.

${ }^{32}$ Now at Max-Planck-Institut für Kernphysik, D-69029 Heidelberg, Germany.

${ }^{33}$ Now at Centro de Investigaciones Energéticas, Medioambientales y Tecnológicas.
}

Inoue et al. 2005; Miniati et al. 2001a, 2001b; Ohno et al. 2002; Miniati 2002, 2003; Sarazin 2002; Brunetti \& Lazarian 2007; Pfrommer et al. 2007, 2008; Pfrommer 2008; Falceta-Goncalves et al. 2010).

Clusters are also home to different types of energetic outflows, and the intracluster medium (ICM) can function as an efficient energy reservoir. Most clusters are seen to harbor radio galaxies around their central regions, whose large, powerful jets of relativistic plasma are interacting vigorously with the ICM (Heinz et al. 1998; Forman et al. 2003; Fabian et al. 2006). A crude estimate of the total energy output by a single powerful radio galaxy is $E_{\mathrm{RG}} \sim\left(10^{60}-10^{62}\right) \mathrm{erg}$, taking reasonable values for the kinetic luminosity $L_{\mathrm{RG}} \sim\left(10^{45}-10^{46}\right) \mathrm{erg} \mathrm{s}^{-1}$ and effective duration of activity $t_{\mathrm{RG}} \sim\left(10^{7}-10^{8}\right)$ yr (McNamara $\&$ Nulsen 2007). The integrated output from the whole cluster radio galaxy population should be even greater (Enßlin et al. 1997, 1998; Inoue \& Sasaki 2001). Although rarely seen in present-day clusters, another source which should have been active in the past are galactic winds, i.e. outflows driven by the joint action of numerous supernovae (Völk et al. 1996). Taking the observed mass of Fe in the ICM to be $M_{\mathrm{Fe}, \text { ICM }} \sim 3 \times\left(10^{9}-\right.$ $\left.10^{10}\right) M_{\odot}$, the energy and $\mathrm{Fe}$ mass ejected by each supernova to be, respectively, $E_{\mathrm{SN}} \sim 10^{51} \mathrm{erg}$ and $M_{\mathrm{Fe}, \mathrm{SN}} \sim 0.1 M_{\odot}$, and an outflow efficiency $\xi_{\mathrm{GW}} \sim 0.1$ (Veilleux et al. 2005), we estimate the total galactic wind energy output to be $E_{\mathrm{GW}} \sim$ $\xi_{\mathrm{GW}} E_{\mathrm{SN}} / M_{\mathrm{Fe}, \mathrm{SN}} M_{\mathrm{Fe}, \mathrm{ICM}} \sim 3 \times\left(10^{60}-10^{61}\right) \mathrm{erg}$. In any case, along with dumping energy, these sources can inject substantial quantities of non-thermal particles into the ICM or could have done so in the past.

Faraday rotation measurements provide a powerful tool to probe the strength of the intracluster magnetic fields (Kim et al. 1991) and even their distribution (Clarke et al. 2001), resulting in the ICM now being known to be permeated by magnetic fields with strengths $B \sim(1-10) \mu \mathrm{G}$ (Carilli \& Taylor 2002; Vogt \& Enßlin 2005), which allow for particle acceleration in shocks up to $\gamma$-ray emitting energies. Observations of radio halos and radio relics have already established that synchrotron emitting electrons with energies reaching $\sim 10 \mathrm{GeV}$ are present in at least some clusters (Feretti 2003; Ferrari et al. 2008), although their precise origin is still unclear. Similar populations of electrons but with harder spectra may produce $\gamma$-rays efficiently via inverse Compton (IC) up-scattering of the cosmic microwave background (Loeb \& Waxman 2000; Totani \& Kitayama 2000; Miniati 2002, 2003; Petrosian et al. 2008). Observations in the hard X-ray regime may suggest the presence of a non-thermal component due to the IC scattering of cosmic microwave photons by relativistic electrons (see Rephaeli et al. 2008 
for a recent review). However, Ajello et al. (2009) found no evidence of a hard tail above the thermal emission in a Swift/Burst Alert Telescope (BAT) sample of clusters. The ICM gas should also provide ample target matter for inelastic collisions leading to pion-decay $\gamma$-rays (Völk et al. 1996; Enßlin et al. 1997; Pfrommer \& Enßlin 2003, 2004a; Pfrommer et al. 2008; Pfrommer 2008) as well as secondary electron injection (Dennison 1980; Vestrand 1982; Blasi \& Colafrancesco 1999; Dolag \& Enßlin 2000; Pfrommer \& Enßlin 2004a; Fujita et al. 2007; Pfrommer 2008). The magnetic fields play another crucial role by confining non-thermal protons within the cluster volume for longer than a Hubble time, i.e. any protons injected into the ICM accumulate throughout the cluster history (Völk et al. 1996; Berezinsky et al. 1997).

Galaxy clusters present very large $\mathrm{M} / \mathrm{L}$ ratios and considerable overdensities, which are crucial for indirect DM searches. Despite the fact that they are not as near as other potential DM candidates, such as the dwarf spheroidal galaxies (Albert et al. 2008d; Aliu et al. 2009a), the large DM masses of clusters could also make them ideal laboratories for the search of a DM annihilation $\gamma$-ray signal (Jeltema et al. 2009; Pinzke et al. 2009).

In this paper, we report the results of the Perseus cluster observation performed by the Major Atmospheric Gamma Imaging Cherenkov (MAGIC) telescope for a total effective time of $24.4 \mathrm{hr}$ during 2008 November and December. In Section 2, we explain the physical motivations for why we chose Perseus over other galaxy clusters and present its main characteristics. In Section 3, we briefly introduce the MAGIC telescope. We then describe the Perseus data sample, the analysis, and the obtained flux upper limits. We discuss the implications for the cosmic ray $(\mathrm{CR})$ pressure and the possible DM annihilation-induced $\gamma$-ray emission in Sections 4 and 5, respectively. In Section 6, we discuss the implications for the jet emission model of the central radio galaxy NGC 1275 . Finally, in Section 7, we summarize our conclusions. All cluster masses and luminosities are scaled to the currently favored value of Hubble's constant $H_{0}=$ $70 \mathrm{~km} \mathrm{~s}^{-1} \mathrm{Mpc}^{-1}$.

\section{TARGET SELECTION AND PRELIMINARIES}

The Perseus cluster, also called A426, is at a distance of 77.7 $\operatorname{Mpc}(z=0.018)$. It is the brightest X-ray cluster (Edge et al. 1992) and hosts a massive cooling flow with high central gas densities of $0.05 \mathrm{~cm}^{-3}$ (see Table 1). Perseus furthermore hosts a luminous radio mini-halo-a diffuse synchrotron emission that fills a large fraction of the cluster core region-and shows a source extension of $\sim 200 \mathrm{kpc}$ (Pedlar et al. 1990). This radio mini-halo is well modeled by the hadronic scenario where the radio emitting electrons are produced in hadronic CR proton interactions with ambient gas protons requiring only a very modest fraction of a few percent $C R$ pressure relative to thermal pressure (Pfrommer \& Enßlin 2004a). In particular, the similarity of the thermal X-ray emission to that of the radio mini-halo comes about naturally as both processes scale with the number density squared. An alternative model for the radio emission has been proposed by Gitti et al. (2002) which explains the radio mini-halo by re-acceleration of relativistic electrons through second-order interactions with magnetohydrodynamic (MHD) turbulence. However, it remains to be shown whether the necessary turbulent energy density can be provided throughout the entire cooling flow region of Perseus. These conditions provide high target densities for hadronic CRp-p interactions and enhance the resulting $\gamma$-ray flux.
The Perseus galaxy cluster was carefully chosen over other nearby clusters after considering the expected $\gamma$-ray emission from the pion-decay and DM annihilation. Moreover, the central radio galaxy NGC 1275 is expected to be a promising GeV-TeV target, and hence is another strong motivation to observe this cluster. In the following subsections, we detail our considerations.

\subsection{Cosmic-Ray-Induced Emission}

In the course of this work, we used cosmological simulations of the formation of galaxy clusters to inform us about the expected spatial and spectral characteristics of the CR-induced $\gamma$-ray emission. A clear detection of the IC emission from shock-accelerated CR electrons will be challenging for Imaging Atmospheric Cherenkov Telescopes (IACTs) due to the large angular extent of these accretion shocks that subtend solid angles corresponding to up to six virial radii. For these instruments, the spatially concentrated pion-decay $\gamma$-ray emission resulting from hadronic CR interactions that dominates the total $\gamma$-ray luminosity (Pfrommer et al. 2008; Pfrommer 2008) should be more readily detectable than the emission from the outer region.

To address the question of universality and predictability of the expected $\gamma$-ray emission, we simulated a sample of 14 galaxy clusters that span one and a half decades in mass and show a variety of dynamical states ranging from relaxed cool core clusters to violent merging clusters (details are given in Section 4.1). In order to find the most promising target cluster in the local Universe for detecting the pion-decay emission, we computed the scaling relations between the $\gamma$-ray luminosity and cluster mass of our sample (Pfrommer 2008) and used these to normalize the CR-induced emission of all clusters in a complete sample of the X-ray brightest clusters (the extended HIFLUGCS catalog; Reiprich \& Böhringer 2002). This favors a high-mass, nearby galaxy cluster with a scaling $M_{200}^{\beta} / D_{\text {lum }}^{2}$, where $M_{200}$ is the virial mass, ${ }^{34} D_{\text {lum }}$ is the luminosity distance, and $\beta \simeq 1.32$ is a weakly model-dependent scaling parameter that provides the rank ordering according to the brightness of each individual cluster (Pfrommer 2008). As a second criterion, we required low zenith angle observations, i.e., below $35^{\circ}$, that ensure the lowest possible energy thresholds and the maximum sensitivity for the detector. We carefully modeled the most promising targets, accounting for the measured gas density and temperatures from thermal X-ray measurements while assuming a constant CR-to-thermal gas ratio (Pfrommer \& Enßlin 2004a). Cluster-wide extended radio synchrotron emission that informs about present high-energy processes was additionally taken into account before we selected the Perseus cluster as our most promising source. Although other clusters showed a somewhat higher $\gamma$-ray flux in our simulations (e.g., Ophiuchus), the facts that Perseus is observable at low zenith angles and that the expected emission is more spatially concentrated make it the best suited target for this observation.

\subsection{Dark Matter Content}

Typically up to $80 \%$ of the total mass of a galaxy cluster is in the form of non-baryonic DM. Since the DM annihilation $\gamma$-ray signal is expected to be proportional to the integrated squared DM density along the line of sight (Evans et al. 2004;

\footnotetext{
34 We define the virial mass $M_{\Delta}$ and the virial radius $R_{\Delta}$ as the mass and radius of a sphere enclosing a mean density that is $\Delta=200$ times the critical density of the Universe.
} 
Table 1

Properties of the Perseus Galaxy Cluster

\begin{tabular}{ccccccc}
\hline \hline$z$ & $D_{\text {lum }}(\mathrm{Mpc})$ & $R_{200}(\mathrm{Mpc})$ & $M_{200}\left(M_{\odot}\right)$ & $L_{X, 0.1-2.4}\left(\mathrm{erg} \mathrm{s}^{-1}\right)$ & $T_{X}(\mathrm{keV})$ & $L_{\nu=1.4}\left(\mathrm{erg} \mathrm{s}^{-1} \mathrm{~Hz}^{-1}\right)$ \\
\hline 0.0183 & 77.7 & 1.9 & $7.71 \times 10^{14}$ & $8.31 \times 10^{44}$ & 6.8 & $3.38 \times 10^{31}$ \\
\hline
\end{tabular}

Notes. Data taken from Reiprich \& Böhringer (2002), Pedlar et al. (1990), and Churazov et al. (2003).

Bergström \& Hooper 2006), it is obvious that galaxy clusters could be good candidates to look for DM as well. This is true despite the fact that they are located at much larger distances than other potential DM candidates, such as dwarf spheroidal galaxy satellites of the Milky Way or the Galactic Center. One obvious reason is the huge amount of DM hosted by clusters compared with the rest of candidates. Perseus, for example, is located $\sim 1000$ times farther than Milky Way dwarfs, but it contains roughly six orders of magnitude more DM than the Willman 1 dwarf galaxy, one of the most promising DM candidates according to recent work (Strigari et al. 2007; Aliu et al. 2009a). Additionally, the presence of substructures could be of crucial importance. Substructures in clusters may significantly enhance the DM signal over the smooth halo, while we do not expect this to be of special relevance for dwarf galaxies since their outer regions are severely affected by tidal stripping (Pinzke et al. 2009; M. A. Sánchez-Conde et al. 2010, in preparation).

Essentially, the annihilation flux is proportional to the product of two parameters (see, e.g., Evans et al. 2004 for details): a first one that captures all the particle physics (DM particle mass, cross section, etc.), which we will label as $f_{\mathrm{SUSY}}$, and a second one, $J_{\text {astro }}$, that accounts for all the astrophysical considerations (DM distribution, telescope point-spread function (PSF), etc.). The particle physics factor just acts as a normalization in the expected annihilation flux, so we can neglect it when performing a comparative study - as we are doing in this section. Concerning the astrophysical factor, the DM distribution is commonly modeled with radial density profiles of the form $\rho(r)=\rho_{s} /\left[\left(r / r_{s}\right)^{\gamma}\left(1+\left(r / r_{s}\right)^{\alpha}\right)^{(\beta-\gamma) / \alpha}\right]$, where $\rho_{s}$ and $r_{s}$ represent a characteristic density and a scale radius, respectively (Kravtsov et al. 1998). These density profiles are well motivated by high-resolution $N$-body cosmological simulations. Here we adopt the Navarro-Frenk-White (Navarro et al. 1997, hereafter NFW) DM density profile, with $(\alpha, \beta, \gamma)=(1,3,1)$. For an NFW profile, $90 \%$ of the DM annihilation flux comes from the region within $r_{s}$ so that the corresponding integrated luminosity is proportional to $r_{s}^{3} \rho_{s}^{2}$. We can derive $r_{s}$ and $\rho_{s}$ for Perseus, assuming $M_{200}=7.7 \times 10^{14} M_{\odot}$ (as given in Table 1) and a concentration of $\sim 6$ (as given by the Bullock et al. 2001 virial mass-concentration scaling relation). We obtain $r_{s}=$ $0.384 \mathrm{Mpc}$ and $\rho_{s}=1.06 \times 10^{15} \mathrm{M}_{\odot} \mathrm{Mpc}^{-3}$, which translates into a total value of $J_{\text {astro }} \sim 1.4 \times 10^{16} \mathrm{GeV}^{2} \mathrm{~cm}^{-5}$ for the scale radius region. In the case of Coma, although slightly $(\sim 15 \%)$ more massive than Perseus, the fact that it is located significantly farther $(101 \mathrm{Mpc})$ translates into a slightly lower annihilation flux. Virgo, only $17 \mathrm{Mpc}$ away from us, gives a larger DM annihilation flux, but here the large extension of the region from which most of the annihilation flux is expected to come compared with Perseus $\left(r_{s} \sim 1.2\right.$ and $r_{s} \sim 0.3$, respectively) could represent an obstacle from the observational point of view. Source extension is of special relevance for single-telescope IACTs, for which point-like sources (sources with an angular extension smaller than or similar to the telescope PSF) are more readily observable.

\subsection{The NGC 1275 Radio Galaxy}

The central NGC 1275 radio galaxy is another strong motivation for $\gamma$-ray observations of the Perseus galaxy cluster. The detection at $\mathrm{TeV}$ energies of the radio galaxies M 87 (Aharonian et al. 2006) and Centaurus A (Aharonian et al. 2009c) has forced a substantial revision of the paradigm whereby very high energy (VHE) emission is a characteristic property of highly relativistic jets closely aligned with the line of sight, establishing radio galaxies as a new class of VHE $\gamma$-ray emitters. Note that NGC 1275 has various characteristics in common with Centaurus $\mathrm{A}$ which has also been interpreted as a possible source of ultra-high-energy CRs (Hardcastle et al. 2009).

The NGC 1275 radio galaxy is the brightest radio source in the northern sky. Its jet inclination angle seems to increase from $10^{\circ}-20^{\circ}$ at milliarcsecond scales up to $40^{\circ}-60^{\circ}$ at arcsecond scales (Dunn et al. 2006). Note that NGC 1275 was classified as a blazar by Angel \& Stockman (1980) because of its optical polarization, and it has been seen to vary in the optical on time scales of a day (Geller et al. 1979). All these elements are promising from the point of view of the $\mathrm{TeV}$ detectability, since they suggest that the emission region is located at the base of the jet. In these conditions, in the scenario based on the structured jet model (Tavecchio \& Ghisellini 2008), we expected VHE emission from the layer of the jet at a level detectable by MAGIC.

\section{MAGIC OBSERVATION AND RESULTS}

The MAGIC telescope is located on the Canary Island of La Palma (2200 m asl, $\left.28^{\circ} .45 \mathrm{~N}, 17.54 \mathrm{~W}\right)$. With a primary mirror diameter of $17 \mathrm{~m}$, it is currently the largest IACT. CRs impinging the Earth atmosphere originate atmospheric showers that in turn produce Cherenkov light. The ultraviolet Cherenkov flashes are reflected in the focal plane of the telescope, where a camera of 577 photomultipliers records the resulting images. MAGIC reconstructs the incoming $\gamma$-ray directions with an accuracy of about 0.1 and achieves an energy resolution above $150 \mathrm{GeV}$ of about 20\% (see Albert et al. 2008c; Aliu et al. 2009b for details).

\subsection{Observation and Analysis}

MAGIC observed the Perseus cluster for $33.4 \mathrm{hr}$ during 2008 November and December, at zenith angles between $12^{\circ}$ and $32^{\circ}$, which guarantees the lowest energy threshold. The observation was performed in the false-source tracking (wobble) mode (Fomin et al. 1994) pointing alternatively to two different sky directions, each at a 0.4 distance from the nominal target position.

The main background for Cherenkov telescopes is due to the hadronic CRs and the night sky background. Our standard analysis procedure is as follows (for a detailed description, see Albert et al. 2008c): data calibration and extraction of the number of photoelectrons per pixel are done (Albert et al. 2008a). This is followed by an image cleaning procedure using the 


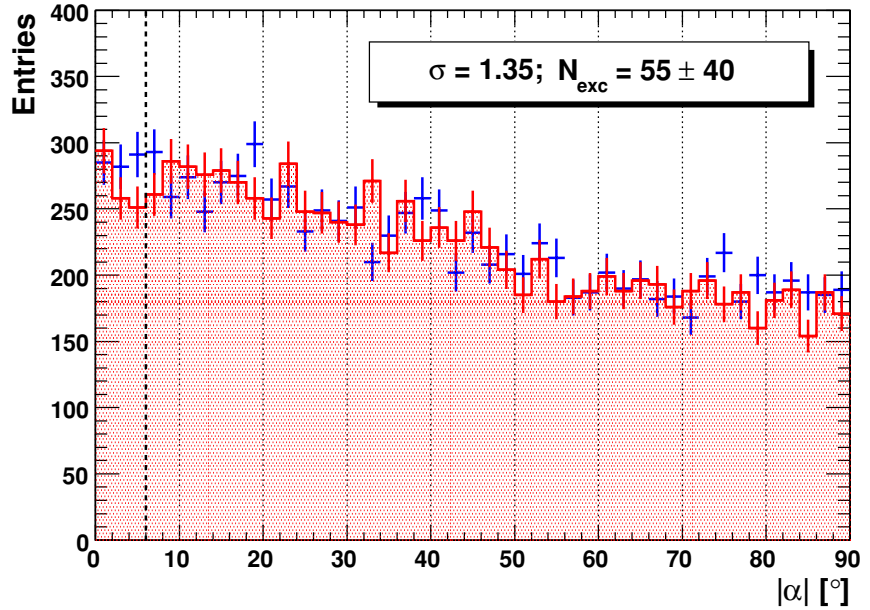

Figure 1. Perseus $\alpha$-plot as seen by MAGIC in $24.4 \mathrm{hr}$ above $250 \mathrm{GeV}$ using a hadronness cut of $<0.05$. The blue crosses represent the signal and the red shaded region is the background. The vertical black dotted line represents the fiducial region $\alpha<6^{\circ}$ where the signal is expected. Only events above $250 \mathrm{GeV}$ are displayed since the best integral sensitivity, around $1.6 \%$ of Crab, is obtained from a Crab Nebula data sample in this energy range.

amplitude and timing information of the calibrated signals. Particularly, the arrival times in pixels containing $>6$ photoelectrons (core pixels) are required to be within a time window of $4.5 \mathrm{~ns}$ and for pixels containing $>3$ photoelectrons (boundary pixels) within a time window of $1.5 \mathrm{~ns}$ from a neighboring core pixel. For the surviving pixels of each event, the shower parameters are reconstructed using the Hillas parameterization algorithm (Hillas 1985). Hadronic background suppression is achieved using a multivariate method called Random Forest (Breiman 2001; Albert et al. 2008b), which uses the Hillas parameters to define an estimator called hadronness (it runs from 0 for gammas to 1 for hadrons) by comparison with Monte Carlo (MC) $\gamma$-ray simulations. Moreover, the Random Forest method is used for the energy estimation of a reconstructed shower. The gamma/hadron $(g / h)$ separation in the analysis was optimized on a sample of well-understood Crab Nebula data, which is commonly accepted as a standard reference source for VHE astronomy.

Part of the data has been rejected mainly due to the bad weather conditions during some observation days. The total data rejected amount to $\sim 27 \%$, resulting in $24.4 \mathrm{hr}$ effective observation time of very high data quality. Independent crosschecks were performed on the data giving compatible results.

\subsection{Results}

Given the good data quality and the low zenith angles of observation, the analysis energy threshold turns out to be $80 \mathrm{GeV}$. Beyond this threshold, no significant excess of $\gamma$-rays above the background was detected in $24.4 \mathrm{hr}$ of observation. In Figure 1, the $\alpha$-plot for energies above $250 \mathrm{GeV}$, where the best integral sensitivity is obtained from a Crab Nebula data sample, is reported. The $\alpha$ parameter is defined as the angular distance between the shower image main axis and the line connecting the observed source position in the camera and the image barycenter. Background events are isotropic in nature and thus produce randomly oriented shower images. This results in a more or less smooth event distribution in the $\alpha$-plot. The $\gamma$-ray events due to the source, on the other hand, are predominantly aligned to the observed position in the camera. For a detected source, this results in a significant excess of events at small $\alpha$. A fiducial

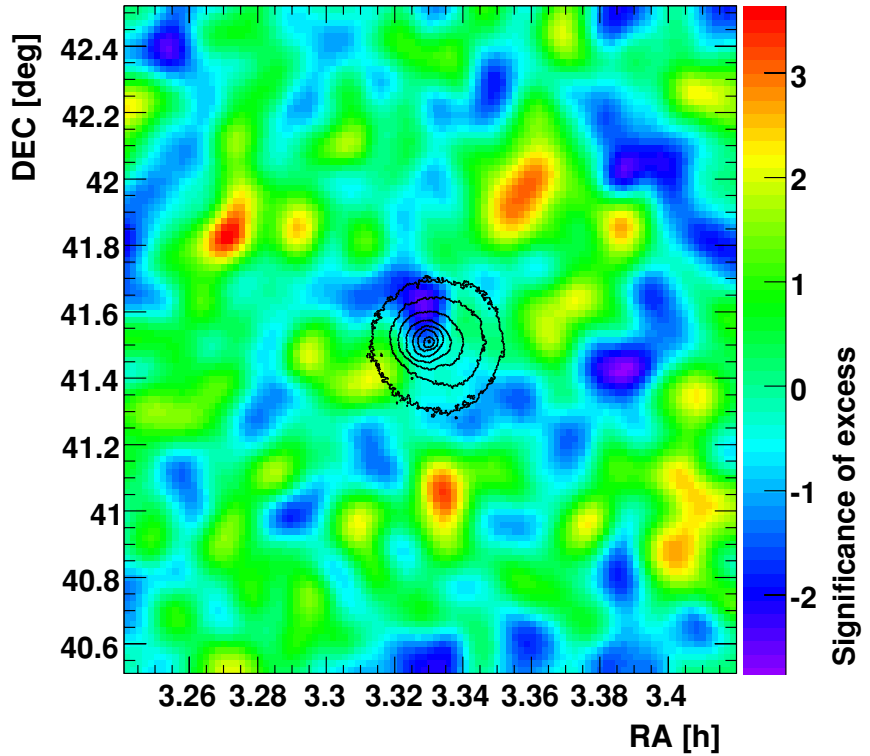

Figure 2. Significance map for events above $150 \mathrm{GeV}$ in the observed Perseus cluster sky region. The significance distribution is consistent with background fluctuations. Black contours from XMM-Newton observations in the X-ray band (Churazov et al. 2003) are also shown. The angular extent of the outermost contours is approximately 0.45 , which corresponds to $\sim 610 \mathrm{kpc}$.

region $\alpha<6^{\circ}$ and a hadronness cut of 0.05 are chosen by optimizing the analysis on a Crab Nebula data sample.

In Figure 2, the significance map for events above $150 \mathrm{GeV}$ in the observed sky region is shown. The source-independent DISP method has been used. This implies the rise of the energy threshold from $80 \mathrm{GeV}$ to around $150 \mathrm{GeV}$ (see Domingo-Santamaria et al. 2005 for a detailed description). The significance distribution in the map is consistent with background fluctuations. In Figure 2, X-ray contours from the XMM-Newton observations (Churazov et al. 2003) are also shown.

The significance was calculated according to Equation (17) of $\mathrm{Li} \& \mathrm{Ma}$ (1983), and upper limit estimation is performed using the Rolke method (Rolke et al. 2005). The upper limits in number of excess events are calculated with a confidence level of $95 \%$. For the upper limit calculation, a systematic uncertainty of $30 \%$ in the energy estimation and effective area calculation is taken into account. Our systematic error budget is obtained by adding up the individual contributions in quadrature. The different sources of systematic uncertainties are mainly related to the differences between the real experimental conditions and the simulated ones (see Albert et al. 2008c for a detailed discussion on the systematic errors). The photon flux upper limit is finally reconstructed for a general $\gamma$-ray spectrum as described in Aliu et al. (2009a).

In Sections 4 and 5, we will discuss the implications of this observation for the CR and DM annihilation-induced $\gamma$-ray flux, respectively. Using the true density profile as obtained by X-ray measurements (Churazov et al. 2003), we will be able to model the spatial characteristics of the CR-induced $\gamma$-ray signal. Our simulations indicate that $60 \%$ of the total $\gamma$-ray flux are contained within a circle of radius $r_{0.6}=0.15$ (this angular scale corresponds to a physical radius of $200 \mathrm{kpc}$ ). We then compare the flux from within this region to the upper limits. As the characteristics of the considered emission region are close to a point source, we use point-like upper limits. The same conclusion is valid also for the DM annihilation signal. In this case, as explained in Section 2.2, $90 \%$ of the expected 
Table 2

Integral Flux Upper Limits Above $100 \mathrm{GeV}$

\begin{tabular}{cc}
\hline \hline$\Gamma$ & $F_{\mathrm{UL}}\left(\times 10^{-12} \mathrm{~cm}^{-2} \mathrm{~s}^{-1}\right)$ \\
\hline-1.5 & 4.63 \\
-2.2 & 6.55 \\
-2.5 & 7.52 \\
\hline
\end{tabular}

Notes. Integral flux upper limits are listed for a power-law $\gamma$-ray spectrum with spectral index $\Gamma$ for energies above $100 \mathrm{GeV}$. The corresponding upper limit for the number of excess events is 186 .

emission is coming from the scale radius region. For Perseus, we obtained $r_{s} \sim 0.3$ which is somewhat extended compared to the telescope angular resolution. However, the fact that the NFW profile is very steep implies that the main DM emission comes from the core of the source that can be considered approximately point-like compared to our angular resolution.

To compute flux upper limits, we assume specific spectral indices that have been motivated by an astrophysical scenario (see the following sections). This "scenario-guided" approach allows us to provide the tightest limits on physically motivated parameters and underlying astrophysical models. In the next sections we will consider flux upper limits computed using a power-law $\gamma$-ray spectrum with spectral indices $\Gamma$ of -1.5 , -2.2 , and -2.5 . In Table 2 , the corresponding integral flux upper limits for energies above $100 \mathrm{GeV}$ are listed.

In Section 4, we will use an integral flux upper limit set above given energy thresholds in order to trace the energy range where we can better constrain the models. In Table 3, the obtained integral flux upper limits for $\Gamma=-2.2$ are shown. Note that we do not compute integral upper limits above $80 \mathrm{GeV}$ (as we have not shown a cumulative $\alpha$-plot for energies above this value). This is because the $g / h$ separation for events below $100 \mathrm{GeV}$ works in a substantially different way with respect to the higher energy events. Therefore, we analyze separately the events below $100 \mathrm{GeV}$ and the events of higher energy, with different sets of analysis cuts.

Finally, for completeness, in Table 4 the differential flux upper limits for the assumed spectral indices are shown in different energy intervals. Spectral energy density (SED) upper limits can also be obtained from those differential flux upper limits, as shown in Section 6 discussing the observation implications for the radio galaxy NGC 1275 .

\subsection{Comparison to Previous Observations}

There are few existing IACT observations of galaxy clusters (Perkins et al. 2006; Perkins 2008; Aharonian et al. 2009a, 2009b; Domainko et al. 2009; Galante et al. 2009; Kiuchi et al. 2009; Acciari et al. 2009). In Section 4.3, we will compare the limits on the CR-to-thermal pressure obtained by other IACTs with those derived in this work. However, there are two observations of the Perseus galaxy cluster made by WHIPPLE (Perkins et al. 2006) and VERITAS (Acciari et al. 2009) with which we can directly compare our upper limits.

The WHIPPLE Collaboration observed the Perseus galaxy cluster (Perkins et al. 2006) for $\sim 13 \mathrm{hr}$ obtaining an integral upper limit above $400 \mathrm{GeV}$ of $4.53 \times 10^{-12} \mathrm{~cm}^{-2} \mathrm{~s}^{-1}$ assuming a spectral index $\Gamma=-2.1$. We can compare this value with our integral upper limit above $400 \mathrm{GeV}$ of $1.83 \times 10^{-12} \mathrm{~cm}^{-2} \mathrm{~s}^{-1}$ with $\Gamma=-2.2$ (see Table 3 ). Our upper limit is significantly lower than the WHIPPLE one; clearly, this is not a surprise as the
Table 3

Integral Flux Upper Limits for a Power-law $\gamma$-ray Spectrum with Spectral Index $\Gamma=-2.2$ Above a Given Energy Threshold $E_{\text {th }}$

\begin{tabular}{cc}
\hline \hline$E_{\mathrm{th}}(\mathrm{GeV})$ & $F_{\mathrm{UL}}\left(\times 10^{-12} \mathrm{~cm}^{-2} \mathrm{~s}^{-1}\right)$ \\
\hline 100 & 6.55 \\
130 & 6.21 \\
160 & 6.17 \\
200 & 5.49 \\
250 & 4.59 \\
320 & 3.36 \\
400 & 1.83 \\
500 & 1.39 \\
630 & 0.72 \\
800 & 0.65 \\
1000 & 0.47 \\
\hline
\end{tabular}

MAGIC telescope belongs to a new generation of IACTs. More recently, the VERITAS Collaboration observed Perseus (Acciari et al. 2009) for $\sim 8 \mathrm{hr}$ and obtained an integral upper limit above $126 \mathrm{GeV}$ of $1.27 \times 10^{-11} \mathrm{~cm}^{-2} \mathrm{~s}^{-1}$ assuming $\Gamma=-2.5$. We can compare this value with our corresponding integral upper limit above $100 \mathrm{GeV}$ of $7.52 \times 10^{-12} \mathrm{~cm}^{-2} \mathrm{~s}^{-1}$ (see Table 2). Despite the fact that the VERITAS sensitivity of about $1 \%$ of Crab Nebula (Otte et al. 2009) is better than the MAGIC one, our upper limit is slightly lower than that found by Acciari et al. (2009) as expected from the significant difference in observation time.

\section{COSMIC-RAY-INDUCED EMISSION}

We use the upper limits on the integrated flux (Table 3) to put constraints on the CR-to-thermal pressure distribution and pursue three different approaches: (1) We perform highresolution hydrodynamical simulations of cluster formation and evolution in a cosmological framework that include CR physics to predict the $\gamma$-ray emission and to obtain limits on the CR-tothermal pressure. (2) Following Pfrommer \& Enßlin (2004a), we use a simplified approach that assumes a constant CR-tothermal energy density, a power-law spectrum in momentum, and compare the resulting CR-to-thermal pressure limits to those obtained by other IACT observations. (3) We use the observed luminosity of the radio mini-halo to place a lower limit on the expected $\gamma$-ray flux in the hadronic model of the radio mini-halo. This translates into a minimum CR pressure that is crucial for disentangling the emission mechanism in the radio and provides a clear prediction for the expected $\gamma$-ray flux.

Before doing so, we detail our cosmological simulations that we base our main analysis on. To this end, we investigated the spatial and spectral properties of $\gamma$-ray emission in these simulations and refer the reader to the theory papers for further details (Pfrommer et al. 2008; Pfrommer 2008; A. Pinzke \& C. Pfrommer 2010, in preparation).

\subsection{Cosmological Simulations}

Simulations were performed using the "concordance" cosmological cold DM model with a cosmological constant $(\Lambda C D M)$ motivated by First Year cosmological constraints of Wilkinson Microwave Anisotropy Probe (WMAP). The cosmological parameters of our model are $\Omega_{\mathrm{m}}=\Omega_{\mathrm{dm}}+\Omega_{\mathrm{b}}=0.3, \Omega_{\mathrm{b}}=0.039$, $\Omega_{\Lambda}=0.7, h=0.7, n=1$, and $\sigma_{8}=0.9$. Here, $\Omega_{\mathrm{m}}$ denotes the total matter density in units of the critical density for geometrical closure today, $\rho_{\text {crit }}(z=0)=3 H_{0}^{2} /(8 \pi G) . \Omega_{\mathrm{b}}$ and $\Omega_{\Lambda}$ denote the densities of baryons and the cosmological constant at the 
Table 4

Differential Flux Upper Limits

\begin{tabular}{cccccccc}
\hline \hline$\Gamma$ & {$[80-100]$} & {$[100-160]$} & {$[160-250]$} & {$[250-400]$} & {$[400-630]$} & {$[630-1000]$} & {$[1000-10000]$} \\
\hline-1.5 & 130.7 & 23.6 & 12.6 & 4.33 & 0.865 & 0.168 & 0.015 \\
-2.2 & 144.8 & 25.3 & 13.2 & 4.53 & 0.897 & 0.174 & 0.018 \\
-2.5 & 150.6 & 25.8 & 13.3 & 4.57 & 0.903 & 0.176 & 0.018 \\
\hline
\end{tabular}

Notes. Differential flux upper limits are listed in units of $10^{-11} \mathrm{~cm}^{-2} \mathrm{~s}^{-1} \mathrm{TeV}^{-1}$ for a power-law $\gamma$-ray spectrum with spectral index $\Gamma$ in energy ranges in units of $\mathrm{GeV}$.

present day, respectively. The spectral index of the primordial power spectrum is denoted by $n$, and $\sigma_{8}$ is the rms linear mass fluctuation within a sphere of radius $8 h^{-1} \mathrm{Mpc}$ extrapolated to $z=0$.

Our simulations were carried out with an updated and extended version of the distributed-memory parallel TreeSPH code GADGET-2 (Springel et al. 2001; Springel 2005). Gravitational forces were computed using a combination of particle-mesh and tree algorithms. Hydrodynamic forces were computed with a variant of the smoothed particle hydrodynamics (SPH) algorithm that conserves energy and entropy where appropriate, i.e. outside of shocked regions (Springel \& Hernquist 2002). We have performed high-resolution hydrodynamic simulations of a sample of galaxy clusters that span over one and a half decades in mass and show a variety of dynamical states ranging from relaxed cool core clusters to violent merging clusters. Our simulated clusters have originally been selected from a low-resolution DM-only simulation (Yoshida et al. 2001). Using the "zoomed initial conditions" technique (Katz \& White 1993), the clusters have been re-simulated with higher mass and force resolution. In high-resolution regions, the DM particles had masses of $m_{\mathrm{dm}}=1.61 \times 10^{9} h_{70}^{-1} M_{\odot}$ and SPH particles $m_{\text {gas }}=2.4 \times 10^{8} h_{70}^{-1} M_{\odot}$, so each individual cluster is resolved by $8 \times 10^{4}-4 \times 10^{6}$ particles, depending on its final mass. The SPH densities were computed from 48 neighbors, allowing the SPH smoothing length to drop at most to half of the value of the gravitational softening length of the gas particles. This choice of the SPH smoothing length leads to our minimum gas resolution of approximately $1.1 \times 10^{10} h_{70}^{-1} M_{\odot}$. For the initial redshift, we chose $1+z_{\text {init }}=60$. The gravitational force softening was of a spline form (e.g., Hernquist \& Katz 1989) with a Plummer-equivalent softening length that is assumed to have a constant comoving scale down to $z=5$ and a constant value of $7 h_{70}^{-1} \mathrm{kpc}$ in physical units at later epochs.

These simulations included radiative hydrodynamics, star formation, and supernova feedback and followed CR physics using a novel formulation that followed the most important injection and loss processes self-consistently while accounting for the CR pressure in the equations of motion (Pfrommer et al. 2006; Enßlin et al. 2007; Jubelgas et al. 2008). To obtain predictions of the $\mathrm{GeV}-\mathrm{TeV} \gamma$-ray emission from clusters, we used an updated version of the CR physics in our code. It is capable of following the spectral evolution of the CR distribution function by tracking multiple CR populations in each gaseous fluid element; each of these populations is described by an amplitude, a low-momentum cutoff, and a characteristic powerlaw distribution in particle momentum with a distinctive slope that is determined by the acceleration process at formation shocks or supernova remnants (A. Pinzke \& C. Pfrommer 2010, in preparation). Adiabatic CR transport processes such as compression and rarefaction and a number of physical source and sink terms which modify the CR pressure of each particle are modeled. The most important sources considered are diffusive shock acceleration at cosmological structure formation shocks and optional injection by supernovae while the primary sinks are thermalization by Coulomb interactions and catastrophic losses by hadronic interactions. We note that the overall normalization of the CR distribution scales with the maximum acceleration efficiency at structure formation shock waves. Following recent observations at supernova remnants (Helder et al. 2009) as well as theoretical studies (Kang \& Jones 2005), we adopt a realistic value of this parameter and assume that $50 \%$ of the dissipated energy at strong shocks is injected into CRs while this efficiency rapidly decreases for weaker shocks (Enßlin et al. 2007).

We computed the $\gamma$-ray emission signal and found that it obeys a universal spectrum and spatial distribution (A. Pinzke \& C. Pfrommer 2010, in preparation). This is inherited from the universal concave spectrum of CRs in galaxy clusters that is caused by the functional form and redshift dependence of the Mach number distribution of structure formation shocks that are responsible for the acceleration of CRs (Pfrommer et al. 2006). The $C R$ distribution has a spectral index of $\Gamma \simeq-2.5$ at $\mathrm{GeV}$ energies and experiences a flattening toward higher energies resulting in $\Gamma \simeq-2.2$ at energies above a few TeV. Hence, the resulting $\gamma$-ray spectrum from CR-induced pion decay shows a characteristic spectral index of $\Gamma \simeq-2.2$ in the energy regime ranging from $100 \mathrm{GeV}$ to $\mathrm{TeV}$. The spatial distribution of the CR number density is mainly governed by adiabatic transport processes (Pfrommer et al. 2007) and similarly attains an approximate universal shape relative to that of the gas density. These findings allow us to reliably model the CR signal from nearby galaxy clusters using their true density profiles as obtained by X-ray measurements that we map onto our simulated density profiles.

In addition to $\mathrm{CR}$ protons, we modeled relativistic electrons that have been accelerated at cosmological structure formation shocks (primary CR electrons) and those that have been produced in hadronic interactions of CRs with ambient gas protons (secondary CR electrons). Both populations of CR electrons contribute to the $\gamma$-ray emission through Compton up-scattering photons from the cosmic microwave background as well as the cumulative starlight from galaxies. It turns out that the piondecay emission of the cluster dominates over the contribution from both IC components-in particular, for relaxed systems (Pfrommer 2008).

In our optimistic CR model (radiative physics with galaxies), we calculated the cluster total $\gamma$-ray flux within a given solid angle. In contrast, we cut the emission from individual galaxies and compact galactic-sized objects in our more conservative model (radiative physics without galaxies). In short, the ICM is a multiphase medium consisting of a hot phase which attained its entropy through structure formation shock waves dissipating 


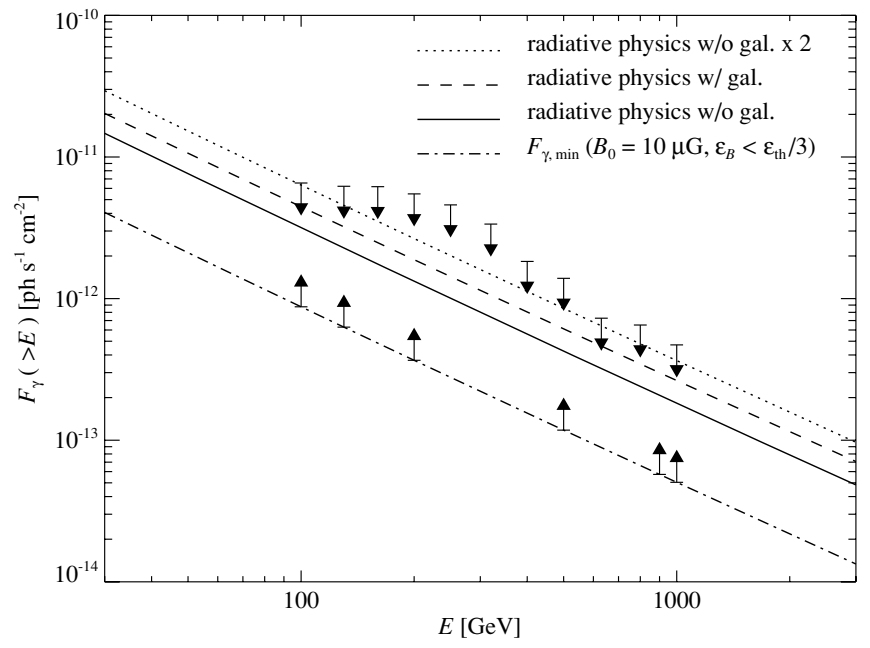

Figure 3. Integral flux upper limits (this work, Table 3) are compared with simulated integrated spectra of the $\gamma$-ray emission from decaying neutral pions that result from hadronic CR interactions with the ambient gas in the Perseus cluster. Our conservative model without galaxies (solid) is contrasted to our model with galaxies (dashed). We scaled our conservative model with a factor of 2 so that it is just consistent with the upper limits obtained in this work (dotted). In our simulations, we assume an observationally motivated large value for the maximum CR energy injection efficiency at structure formation shocks and convert half of the dissipated energy to CRs at strong shocks. Smaller values would imply smaller $\gamma$-ray fluxes. Additionally shown are minimum $\gamma$-ray flux estimates for the hadronic model of the radio mini-halo of the Perseus cluster (dash-dotted with minimum flux arrows; see the main text for details). Note that a non-detection of $\gamma$-rays at this level seriously challenges the hadronic model.

gravitational energy associated with hierarchical clustering into thermal energy. The dense, cold phase consists of the true interstellar medium (ISM) within galaxies and at the cluster center as well as the ram-pressure-stripped ISM. These cold dense gas clumps dissociate incompletely in the ICM due to insufficient numerical resolution as well as so far incompletely understood physical properties of the cluster plasma. All of these phases contribute to the $\gamma$-ray emission from a cluster. To assess the bias associated with this issue, we performed our analysis with both limiting cases bracketing the realistic case.

In Figure 3, we compare the integral flux upper limits obtained in this work (see Table 3) with the simulated flux that is emitted within a circle of radius $r_{0.6}=0.15$ for our two models, with and without galaxies. The upper limits are a factor of 2 larger than our conservative model and a factor of 1.5 larger than our most optimistic model predictions implying consistency with our cosmological cluster simulations. We note however that our simulated flux represents a theoretical upper limit of the expected $\gamma$-ray flux from structure formation CRs; lowering the maximum acceleration efficiency would decrease the CR number density as well as the resulting $\gamma$-ray emission.

\subsection{Constraints on the Cosmic Ray Pressure}

In Figure 4, we show the simulated $\gamma$-ray surface brightness map of a cooling flow cluster of mass similar to Perseus. As the CR-induced $\gamma$-ray flux is a radially declining function, so is the CR pressure. A quantity that is of great theoretical interest is the $\mathrm{CR}$ pressure relative to the thermal pressure $X_{\mathrm{CR}}=P_{\mathrm{CR}} / P_{\mathrm{th}}$, as it directly assesses the $\mathrm{CR}$ bias of hydrostatic cluster masses since the $\mathrm{CR}$ pressure enters in the equation of motion. On the right-hand side of Figure 4, we show the profile of the CR-to-thermal pressure (volume-weighted) of this simulated cluster. Moving from the periphery toward the center, this quantity is a steadily declining function until we approach the cooling flow region around the $\mathrm{cD}$ galaxy of this cluster (similar to NGC 1275) where the CR pressure rises dramatically relative to that of the thermal gas which cools on a short time scale (Pfrommer et al. 2006). The volume average is $\left\langle X_{\mathrm{CR}}\right\rangle=\left\langle P_{\mathrm{CR}}\right\rangle /\left\langle P_{\mathrm{th}}\right\rangle=0.02$, dominated by the region around the virial radius, while the ratio of $\mathrm{CR}$-tothermal energy is given by $E_{\mathrm{CR}} / E_{\mathrm{th}}=0.032 .{ }^{35}$ Perseus has a smaller mass and a corresponding temperature that is only half of that of our simulated cooling flow cluster. Noting that $X_{\mathrm{CR}} \propto 1 / P_{\text {th }} \propto 1 / k T,{ }^{36}$ we expect these values to be a factor of $\simeq 2$ larger in Perseus, yielding $\left\langle X_{\mathrm{CR}}\right\rangle \simeq 0.04$ for the entire cluster and $\left\langle X_{\mathrm{CR}}\right\rangle \simeq 0.02$ for the core region that we probe with the present observation.

We have to scale our conservative model prediction by a factor of $\sim 2$ to reach the upper limits (cf. Figure 3 ) which implies that this work constrains the relative pressure contained in CRs to $<8 \%$ for the entire cluster and to $<4 \%$ for the cluster core region. The presence of dense gas clumps potentially biases the simulated $\gamma$-ray flux high and hence the inferred limits on $X_{\mathrm{CR}}$ low. Another source of bias could be unresolved point sources inside the cluster such as an active galactic nucleus (AGN). In the presented simulation of the cool core cluster $\mathrm{g} 51$, the bias due to subclumps amounts to a factor of 1.5 but it could be as high as 2.4 which is the mean difference between our conservative and optimistic models across our scaling relations. We note however that the latter case is already excluded by our upper limits provided the maximum shock acceleration efficiency is indeed as high as $50 \%$. While there are indications from supernova remnant observations of one rim region (Helder et al. 2009) as well as theoretical studies (Kang \& Jones 2005) that support such high efficiencies, to date it is not clear whether these efficiencies apply in an average sense to strong collisionless shocks or whether they are realized for structure formation shocks at higher redshifts. Improving the sensitivity of the presented type of observations will help in answering these profound plasma astrophysics questions.

In Figure 4, we additionally compare a simulation where we only accelerate CRs at structure formation shocks with one where we additionally account for CRs that are injected through supernova feedback within the star-forming regions in our simulation. Outside the $\mathrm{cD}$ galaxy, there is no significant difference visible which suggests that the CRs injected into the ICM by supernova-driven winds are negligible compared with those accelerated by structure formation shocks. While this is partly an artifact of our simulations that neglect CR diffusion, we expect this behavior due to the adiabatic losses that CRs suffer as they expand from their compact galactic ISM into the dilute ICM. Assuming a conservative value for the density contrast of $\Delta=10^{-3}$, the CR pressure is diluted by $P_{\mathrm{CR}} \sim \Delta^{4 / 3} P_{\mathrm{CR}, \mathrm{ISM}} \sim 10^{-4} P_{\mathrm{CR}, \mathrm{ISM}}$.

\footnotetext{
35 Note that for a CR population in clusters that have been accelerated in structure formation shocks, the relativistic limit $E_{\mathrm{CR}} / E_{\mathrm{th}}=2\left\langle P_{\mathrm{CR}}\right\rangle /\left\langle P_{\mathrm{th}}\right\rangle$ is not applicable since the $\mathrm{CR}$ pressure is dominated by the transrelativistic regime. This implies a somewhat harder equation of state for the CRs with a larger adiabatic index and yields the relation $E_{\mathrm{CR}} / E_{\mathrm{th}}=1.6\left\langle P_{\mathrm{CR}}\right\rangle /\left\langle P_{\mathrm{th}}\right\rangle$.

36 This relation should only hold for regions with long thermal cooling times compared to the dynamical time scale. In particular, it breaks down toward the center of a cooling flow cluster where the thermal gas cools on a shorter time scale such that the forming cooling flow causes adiabatic contraction of the $\mathrm{CR}$ population.
} 

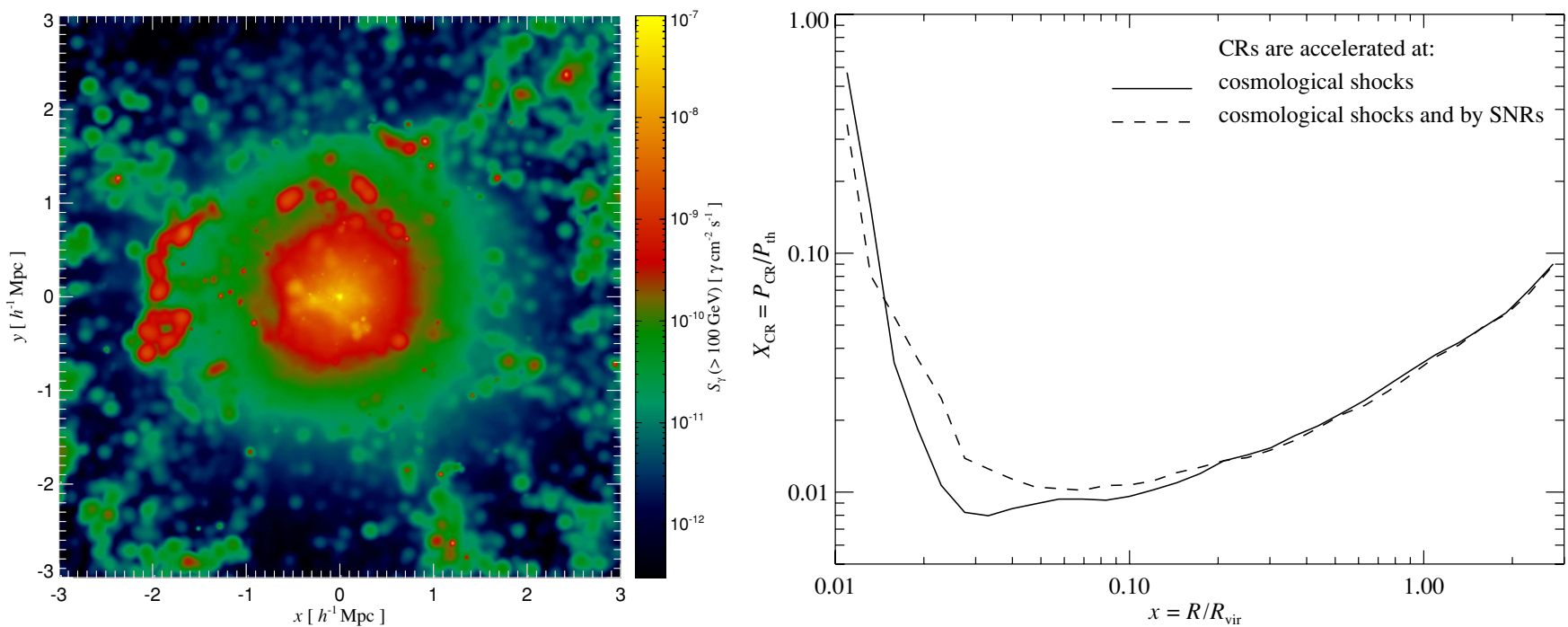

Figure 4. Left: simulated $\gamma$-ray emission at energies $E>100 \mathrm{GeV}$ from a cluster that has twice the mass as Perseus (using the simulation of the cooling flow cluster g51 from Pfrommer et al. 2008). We show the sum of pion-decay-induced $\gamma$-rays (which dominates the central and the total flux) and the IC emission of CR electrons accelerated at formation shocks and by hadronic CR interactions. Right: profile of the CR-to-thermal pressure (volume-weighted) of this cluster. We contrast a simulation where we only accelerate CRs at structure formation shocks of the entire cosmic history (solid) with one where we additionally account for CRs that are injected through supernova feedback within the star-forming regions in our simulation (dashed).

\subsection{Simplified Approach and Comparison to Previous Results}

As anticipated in Section 3.3, there are few existing IACT observations of galaxy clusters, some of which derived limits on the CR-to-thermal pressure contained in clusters, in particular the WHIPPLE observation of the Perseus cluster (Perkins et al. 2006) and the HESS observations of the Abell 85 (Aharonian et al. 2009a; Domainko et al. 2009) and Coma (Aharonian et al. $2009 b$ ) clusters. These works used simplifying assumptions about the spectral and spatial distribution of CRs. They typically assumed a single CR power-law distribution with a spectral index of $\Gamma=-2.1$ (that provides optimistic limits on the CRto-thermal pressure) and assumed that the CR energy density is a constant fraction of the thermal energy density throughout the entire cluster. Based on these two assumptions, WHIPPLE and HESS found in Perseus and Abell $85 E_{\mathrm{CR}} / E_{\mathrm{th}}<0.08$, respectively, while HESS found $E_{\mathrm{CR}} / E_{\mathrm{th}}<0.2$ in Coma.

To facilitate comparison with these earlier works, we repeated the data analysis with a spectral index $\Gamma=-2.1$ to obtain an integral upper limit $\mathcal{F}_{\mathrm{UL}}(>100 \mathrm{GeV})=6.22 \times 10^{-12} \mathrm{~cm}^{-2} \mathrm{~s}^{-1}$. Following the formula of Pfrommer \& Enßlin (2004a), we compute the $\gamma$-ray flux of a CR population with $\Gamma=-2.1$ within a circular region of radius $r_{0.6}=0.15$ or equivalently $200 \mathrm{kpc}$. In our isobaric model of CRs, we assume that the CR pressure scales exactly as the thermal pressure and constrain $E_{\mathrm{CR}} / E_{\mathrm{th}}<0.053$ which corresponds to an averaged relative pressure of $\left\langle X_{\mathrm{CR}}\right\rangle=\left\langle P_{\mathrm{CR}}\right\rangle /\left\langle P_{\mathrm{th}}\right\rangle=0.033$. This would be the most stringent upper limit on the CR energy in a galaxy cluster.

In our adiabatic model of $C R s$, we account for the centrally enhanced CR number density due to adiabatic contraction during the formation of the cooling flow (Pfrommer \& Enßlin 2004a). We assume that the CRp population scaled originally as the thermal population but was compressed adiabatically during the formation of the cooling flow without relaxing afterward (we adopted temperature and density profiles given by Churazov et al. 2003). In this model, we obtain an enhanced $\gamma$-ray flux level for virtually the same volume-averaged CR pressure or vice versa for a given flux limit; hence, we can put a tighter constraint on the averaged $\mathrm{CR}$ pressure. We constrain $E_{\mathrm{CR}} / E_{\mathrm{th}}<0.03$ which corresponds to an averaged relative pressure of $\left\langle X_{\mathrm{CR}}\right\rangle=\left\langle P_{\mathrm{CR}}\right\rangle /\left\langle P_{\mathrm{th}}\right\rangle=0.019$.

How can we reconcile these tighter limits with our simulationbased slightly weaker limit? We have to compare our simulated $\mathrm{CR}$ profile to a CR distribution that does not show any enhancement relative to the gas density. In the central region for $r<200 \mathrm{kpc}$, we derive an adiabatic compression factor of 1.7 that matches that in our simplified approach — suggesting that our simple adiabatic model captures the underlying physics quite realistically. Second, we have then to relate the pressure of a power-law spectrum with $\Gamma=2.1$ to our simulated concave spectrum. Noting that the $\gamma$-rays at $100 \mathrm{GeV}$ are produced by CR protons at $\simeq 1 \mathrm{TeV}$, we normalize both spectra at $1 \mathrm{TeV}$ and find that the simulated spectrum contains a larger pressure by a factor of 1.8. This factor brings the limit of our simplified adiabatic model into agreement with our simulation-based limit of the relative CR pressure $\left\langle X_{\mathrm{CR}}\right\rangle<4 \%$ for the cluster core region. Finally, since $\gamma$-ray observations are only sensitive to the cluster core regions (the emission is expected to peak in the center due to the high target gas densities), they cannot constrain the average CR-to-thermal pressure within the entire cluster. Hence, we have to use cosmological cluster simulations to address how much CR-to-thermal pressure could be additionally hidden in the peripheral cluster regions.

\subsection{Minimum $\gamma$-ray Flux}

For clusters that host radio (mini-)halos, we are able to derive a minimum $\gamma$-ray flux in the hadronic model of CR interactions. The idea is based on the fact that a steady-state distribution of $\mathrm{CR}$ electrons loses all its energy to synchrotron radiation for strong magnetic fields $\left(B \gg B_{\mathrm{CMB}} \simeq 3.2 \mu \mathrm{G}\right.$ ) so that the ratio of $\gamma$-ray to synchrotron flux becomes independent of the spatial distribution of CRs and thermal gas (Pfrommer 2008). This can be easily seen by considering the pion-decay-induced $\gamma$-ray luminosity $L_{\gamma}$ and the synchrotron luminosity $L_{v}$ of a steadystate distribution of $\mathrm{CR}$ electrons that has been generated by 
hadronic CR interactions:

$$
\begin{gathered}
L_{\gamma}=A_{\gamma} \int d V n_{\mathrm{CR}} n_{\mathrm{gas}}, \\
L_{v}=A_{v} \int d V n_{\mathrm{CR}} n_{\mathrm{gas}} \frac{\varepsilon_{B}^{\left(\alpha_{v}+1\right) / 2}}{\varepsilon_{\mathrm{CMB}}+\varepsilon_{B}} \\
\simeq A_{v} \int d V n_{\mathrm{CR}} n_{\mathrm{gas}} \quad \text { for } \varepsilon_{B} \gg \varepsilon_{\mathrm{CMB}} .
\end{gathered}
$$

Here $A_{\gamma}$ and $A_{\nu}$ are dimensional constants that depend on the hadronic physics of the interaction (Pfrommer et al. 2008; Pfrommer 2008) and $\alpha_{v} \simeq 1$ is the observed synchrotron spectral index. Hence, we can derive a minimum $\gamma$-ray flux in the hadronic model:

$$
\mathcal{F}_{\gamma, \text { min }}=\frac{A_{\gamma}}{A_{v}} \frac{L_{v}}{4 \pi D_{\text {lum }}^{2}},
$$

where $L_{v}$ is the observed luminosity of the radio mini-halo and $D_{\text {lum }}$ denotes the luminosity distance to the respective cluster. Lowering the magnetic field would require an increase in the energy density of CR electrons to reproduce the observed synchrotron luminosity and thus increase the associated $\gamma$-ray flux.

Using the values of Table 1 , we obtain a minimum $\gamma$ ray flux in the hadronic model of the radio mini-halo of $\mathcal{F}_{\gamma, \min }(>100 \mathrm{GeV})=6 \times 10^{-13} \mathrm{~cm}^{-2} \mathrm{~s}^{-1}$, assuming a powerlaw $\mathrm{CR}$ distribution with $\Gamma \gtrsim-2.3$. This lower limit is independent of the spatial distribution of CRs and magnetic fields. We note that the spectral index is consistent with the radio data. ${ }^{37}$ It turns out that the requirement of strong magnetic fields violates the energy conditions in clusters as it implies a magnetic energy density that is larger than the thermal energy density-in particular, at the peripheral cluster regions. The minimum $\gamma$-ray flux condition requires a constant (large) magnetic field strength throughout the cluster while the thermal energy density is decreasing by more than a factor of 100 from its central value. This would imply that the magnetic field eventually dominates the energy density at the virial regions - a behavior that is unstable as it is subject to Parker-like buoyancy instabilities. Additionally, such a configuration would be impossible to achieve in the first place as the magnetic energy density typically saturates at a fixed fraction of the turbulent energy density which itself is only a small fraction of the thermal energy density in clusters (Schuecker et al. 2004). Hence, these considerations call for lowering the assumed cluster magnetic fields which should strengthen the lower limits on the $\gamma$-ray flux considerably - however at the expense that these limits inherit a weak dependence on the spatial distribution of magnetic fields and CRs.

Estimates of magnetic fields from Faraday rotation measures (RMs) have undergone a revision in the last few years with more recent estimates typically in the order of a few $\mu \mathrm{G}$ with slightly higher values up to $10 \mu \mathrm{G}$ in cooling flow clusters (Clarke 2004; Enßlin \& Vogt 2006). For the Perseus radio mini-halo, Faraday RMs are available only on very small scales (Taylor et al. 2006), i.e. few tens of pc. RM estimates are of the order of $\sim 7000 \mathrm{rad} \mathrm{m}^{2}$ leading to magnetic field values of $\sim 25 \mu \mathrm{G}$

\footnotetext{
37 The CR protons responsible for the $\mathrm{GHz}$ radio emitting electrons are $\sim 100$ times less energetic than those $\mathrm{CR}$ protons that are responsible for the $\mathrm{TeV}$ $\gamma$-ray emission. This is consistent with the concave curvature found in the CR spectrum by A. Pinzke \& C. Pfrommer (2010, in preparation).
}

assuming that the Faraday screen is localized in the ICM. This, however, appears to be unlikely as variations of $10 \%$ in the RM are observed on pc scales (Taylor et al. 2002), while ICM magnetic fields are expected to be ordered on significantly larger scales of a few kpc (Taylor et al. 2006; Vogt \& Enßlin 2005; Enßlin \& Vogt 2006). Application of the classical minimumenergy argument to the Perseus radio mini-halo data leads to estimates for the central magnetic field strength of $B_{0} \simeq 7 \mu \mathrm{G}$ or even $B_{0} \simeq 9 \mu \mathrm{G}$ for the more appropriate hadronic minimumenergy argument (Pfrommer \& Enßlin 2004b).

We select a cooling flow cluster of our sample that is morphologically similar to Perseus with a mass $M_{200} \simeq 10^{15} M_{\odot}$ (the simulated cluster g51 of Pfrommer et al. 2008). We adopt a conservative choice for the central magnetic field strength of $\sim 10 \mu \mathrm{G}$ and parameterize the magnetic energy density in terms of the thermal energy density by $\varepsilon_{B} \propto \varepsilon_{\mathrm{th}}^{0.5}$ which ensures $\varepsilon_{B}<\varepsilon_{\text {th }} / 3$ in the entire cluster. This allows us to strengthen the physically motivated lower limit to $\mathcal{F}_{\gamma \text {,phys. min }}(>100 \mathrm{GeV})=$ $8.5 \times 10^{-13} \mathrm{~cm}^{-2} \mathrm{~s}^{-1}$ as shown by the dash-dotted line in Figure 3. In the hadronic model, this minimum $\gamma$-ray flux implies a minimum CR pressure relative to the thermal pressure. Figure 3 shows that the minimum flux $\mathcal{F}_{\gamma \text {,phys. min }}$ is a factor of 3.6 lower than the simulated flux for Perseus in our conservative model. As seen in Section 4.2, this model corresponds to a relative CR pressure of $\left\langle X_{\mathrm{CR}}\right\rangle=\left\langle P_{\mathrm{CR}}\right\rangle /\left\langle P_{\mathrm{th}}\right\rangle=$ 0.04 where the averages represent volume averages across the entire cluster. Hence we obtain a minimum relative CR pressure, $\left\langle X_{\mathrm{CR}, \min }\right\rangle=\left\langle P_{\mathrm{CR}, \min }\right\rangle /\left\langle P_{\mathrm{th}}\right\rangle / 3.6=0.01$. This minimum $\mathrm{CR}$ pressure corresponds to a minimum total $\mathrm{CR}$ energy of $E_{\mathrm{CR} \text { min }}=E_{\mathrm{CR} \text { min }} / E_{\mathrm{th}} \times E_{\mathrm{th}}=1.6\left\langle X_{\mathrm{CR}, \min }\right\rangle \times E_{\mathrm{th}}=$ $9 \times 10^{61} \mathrm{erg}$ where we integrated the temperature and density profiles from X-ray observations (Churazov et al. 2003) to obtain the total thermal energy of $E_{\mathrm{th}}=5.7 \times 10^{63} \mathrm{erg}$. These considerations show the huge potential of combining future $\mathrm{TeV}$ $\gamma$-ray and radio observations in constraining physical models of the non-thermal cluster emission and of obtaining important insights into the average distribution of cluster magnetic fields.

\section{DARK MATTER ANNIHILATION}

As discussed in Section 2.2, the expected DM annihilation flux is proportional to the product of a factor that encloses all the particle physics and a second one that accounts for all the involved astrophysics. Therefore, in order to obtain an estimate of the annihilation flux, we need to choose a particular particle physics model (that was not needed in Section 2.2, since only a comparative study was done there) in addition to the modeling of the DM distribution. Although the uncertainties in the particle physics factor $f_{\text {SUSY }}$ are very large and spread over some orders of magnitude (see, e.g., Albert et al. 2008d), it is common to use the most optimistic value for a given energy threshold of the telescope. This factor just acts as a rescaling factor in the total flux, so we could change to the other particle physics model simply by rescaling for its new value. Let us assume $f_{\mathrm{SUSY}}=10^{-32} \mathrm{GeV}^{-2} \mathrm{~cm}^{3} \mathrm{~s}^{-1}$ above $100 \mathrm{GeV}$, which corresponds to one of the most optimistic allowed scenarios at the energies of interest here (Sánchez-Conde et al. 2007), with the neutralino as a DM particle. Then, taking a value of $1.4 \times 10^{16} \mathrm{GeV}^{2} \mathrm{~cm}^{-5}$ for the integrated astrophysical factor inside $r_{s}$ (as given in Section 2.2), we obtain a maximum DM annihilation flux of $\sim 1.4 \times 10^{-16} \mathrm{~cm}^{-2} \mathrm{~s}^{-1}$ for energies above $100 \mathrm{GeV}$. The comparison with the derived upper limits from our observations is not very constraining. Assuming a generic 
DM annihilation spectrum without a cutoff and a spectral index of -1.5 as a good approximation (e.g., Albert et al. 2008d; Aliu et al. 2009a), it can be seen from Table 2 that we need a boost in flux in the order of $10^{4}$ to reach the predicted DM annihilation flux values, since $\mathcal{F}_{\mathrm{UL}}(>100 \mathrm{GeV})=4.63 \times 10^{-12} \mathrm{~cm}^{-2} \mathrm{~s}^{-1}$.

This boost factor could come from different uncertainties that may enhance the annihilation $\gamma$-ray flux notably and that were not taken into account in the above calculation. One of them, the presence of substructures, could play a crucial role for Perseus, as explained in Section 2.2. Although still uncertain, its effect could enhance the expected annihilation flux by more than a factor of 10 for Perseus-size halos according to Kuhlen et al. (2008). More recent work has shown that the expected boost factors could be as high as 200 (Springel et al. 2008a, $2008 \mathrm{~b}$ ). However, with IACTs it is challenging to make use of these large boost factors as their contribution is expected to be more important on large angular scales comparable to the virial extent of the cluster. Detailed modeling of the substructures is needed in order to correctly evaluate their impact on the Perseus DM-induced signal. Finally, recently proposed mechanisms on the particle physics side, such as the internal bremsstrahlung (Bringmann et al. 2008) and the Sommerfeld effect (Lattanzi \& Silk 2009; Pinzke et al. 2009), could also enhance the DM annihilation flux by more than one order of magnitude for some particle physics models.

It is worth noting that the result obtained here for the boost factor needed in order to probe the predicted DM annihilation flux is comparable with previous observations of the Milky Way satellite galaxies (Albert et al. 2008d; Aliu et al. 2009a).

\section{THE NGC 1275 EMISSION}

The SED of the NGC 1275 core is shown in Figure 5. The radio and optical data represented with gray filled circles (Abdo et al. 2009) have been obtained with low resolution and thus include a large contribution from the large-scale regions of the jet (radio) and from the host galaxy (optical). In the following, we model the data corresponding to the core emission. This is different from what was done by Abdo et al. (2009) who used the low resolution data in their models. We calculated our upper limit, shown in Figure 5 as a red arrow, assuming that the spectrum in the MAGIC energy band is a power law with spectral index $\Gamma=-2.5$, as indicated by the extrapolation of the last points of the Fermi-LAT spectrum. Note, however, that the level of the differential upper limits is only weakly dependent on the assumed spectral index (see Table 4).

The data clearly show a double-peak SED. The radio-optical data suggest a peak of the emission in the IR band, similar to the case of other $\gamma$-ray emitting radio galaxies (Tavecchio \& Ghisellini 2008, 2009). High-energy data constrain the peak frequency of the second component at about $100 \mathrm{MeV}$. As discussed in Tavecchio \& Ghisellini (2008), in these cases a one-zone synchrotron self-Compton (SSC) model for the entire emission is implausible, since the large separation in frequency between the two peaks would require extremely large values of the Doppler factor:

$$
\delta \simeq 258 L_{\mathrm{s}, 42.8} L_{\mathrm{C}, 43.4}^{-1 / 2} \nu_{\mathrm{s}, 13.5}^{-2} \nu_{\mathrm{C}, 23} R_{16}^{-1},
$$

where $L_{\mathrm{s}}=v_{\mathrm{s}} L\left(v_{\mathrm{s}}\right), L_{\mathrm{C}}=v_{\mathrm{c}} L\left(v_{\mathrm{c}}\right), v_{\mathrm{s}}$ and $v_{\mathrm{C}}$ are the synchrotron and SSC peak luminosities and frequencies, respectively, and $R$ is the size of the emitting region. Here $Q=10^{x} Q_{x}$ in cgs units, and we use the values derived for NGC 1275. In

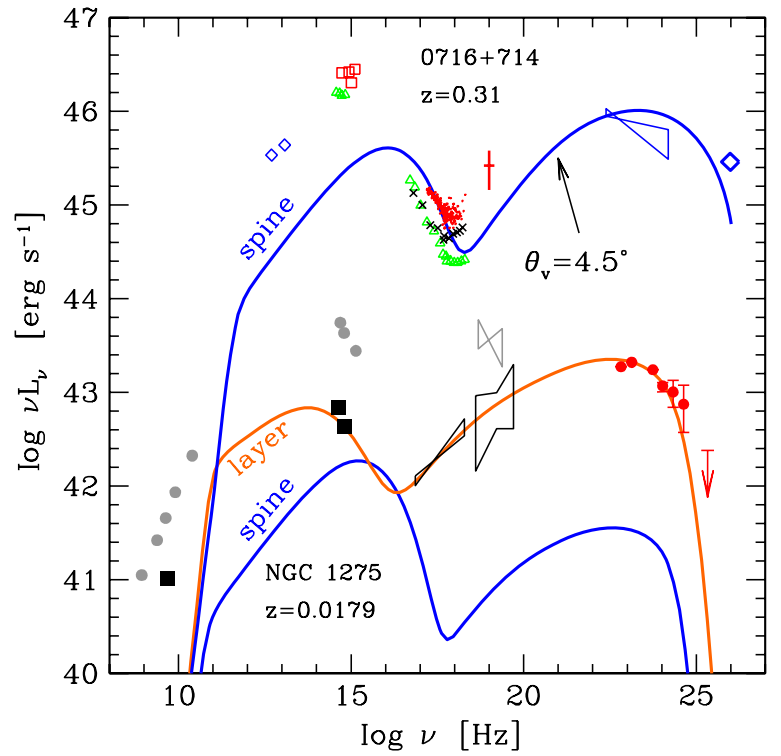

Figure 5. SED of the NGC 1275 core (lower two lines and data) and that of the well-known blazar S5 0716+714 for comparison (upper line and data). Gray filled circles are data points in the radio and optical bands from Abdo et al. (2009). Filled black squares show, instead, the radio (VLBI; Taylor et al. 1996) and the optical emission (Hubble Space Telescope (HST); Chiaberge et al. 1999) of the core alone. The soft X-ray bow tie is from Chandra (Balmaverde et al. 2006), while the red filled circles represent the Fermi-LAT spectrum taken from Abdo et al. (2009). The red arrow shows the MAGIC upper limit between 80 and $100 \mathrm{GeV}$. The lower blue and red lines show the emission from the spine and the layer of the structured jet, respectively. The upper blue line is the SED of the spine as observed at a small angle (see the text for details); for comparison, we report historical data of S5 0716+714 (data from Tavecchio \& Ghisellini 2009 and references therein).

this estimate, we assume the typical size of the emission regions derived in blazars, $R=10^{16} \mathrm{~cm}$, though the Fermi-LAT data do not allow us to constrain the radius of the emission region using the variability (Abdo et al. 2009). Such large values of the Doppler factor are rather unlikely. Typical values found modeling the SED of blazars are around 10-20 (e.g., Celotti \& Ghisellini 2008), with few extreme TeV BL Lacs requiring larger values during exceptional states $(\delta \sim 50-100$; e.g., Begelman et al. 2008; Ghisellini \& Tavecchio 2008). Arguments based on the observation of superluminal motions at Very Long Baseline Interferometry (VLBI) scales (e.g., Kellermann et al. 2004) and on the unification of blazars with radio galaxies also require values around 10 (e.g., Urry \& Padovani 1995).

The most direct way to overcome the problem posed by the large Doppler factor is to assume two emission regions, as in the spine-layer model of Ghisellini et al. (2005). In this scenario the jet is assumed to be structured, with a fast inner region (the spine) surrounded by a slower sheet (the layer). Both components produce synchrotron and IC radiation and they are in radiative interplay: the synchrotron radiation from one component is seen boosted (by the relative velocity) by the other one and thus the IC emission of both regions is enhanced with respect to the standard SSC. In radio galaxies, in which the jet is observed at relatively large angles, the emission is expected to be dominated by the layer, which, due to the lower bulk Lorentz factor, has a larger emission cone. At a smaller angle, instead, the emission is dominated by the spine, as in blazars.

We reproduce the SED with the spine-layer model. The orange line in Figure 5 shows the emission from the layer, while the spine produces the emission shown by the blue bottom line. 
The spine is assumed to be a cylinder of radius $R=1.5 \times$ $10^{16} \mathrm{~cm}$, height $H_{\mathrm{S}}=1.5 \times 10^{16} \mathrm{~cm}$ (as measured in the spine frame), and in motion with bulk Lorentz factor $\Gamma_{\mathrm{S}}=15$. The layer is modeled as a hollow cylinder with internal radius $R$, external radius $R_{2}=1.2 \times R$, height $H_{\mathrm{L}}=4 \times 10^{16} \mathrm{~cm}$ (as measured in the frame of the layer), and bulk Lorentz factor $\Gamma_{\mathrm{L}}=3$. Each region contains a tangled magnetic field with intensity $B_{\mathrm{S}}=2.5 \mathrm{G}$ and $B_{\mathrm{L}}=1 \mathrm{G}$, and it is filled by relativistic electrons assumed to follow a (purely phenomenological) smoothed broken power-law distribution extending from $\gamma_{\min }$ to $\gamma_{\max }$ and with indices $n_{1}, n_{2}$ below and above the break at $\gamma_{\mathrm{b}}$. For the spine we use $\gamma_{\min }=40$, $\gamma_{\mathrm{b}}=2 \times 10^{4}, \gamma_{\max }=10^{5}, n_{1}=2, n_{2}=3.5$. For the layer $\gamma_{\min }=10, \gamma_{\mathrm{b}}=4 \times 10^{3}, \gamma_{\max }=10^{5}, n_{1}=2.4$, $n_{2}=4.2$. The normalization of these distributions is calculated assuming that the systems produce an assumed (bolometric) synchrotron luminosity $L_{\mathrm{syn}, \mathrm{S}}^{\prime}=10^{42} \mathrm{erg} \mathrm{s}^{-1}$ and $L_{\mathrm{syn}, \mathrm{L}}^{\prime}=2.7 \times$ $10^{41} \mathrm{erg} \mathrm{s}^{-1}$ (as measured in the local comoving frame of the spine and layer, respectively), which is an input parameter of the model. As said above, the seed photons for the IC scattering are not only those produced locally in the spine (layer), but we also consider the photons produced in the layer (spine). We assume a viewing angle of $\theta=15^{\circ}$. As discussed above, the same jet observed at a smaller angle would be dominated by the emission from the spine and we expect that its SED resembles those of typical blazars. We show the SED of the jet when observed at an angle of 4.5 (blue upper line in Figure 5). The SED is dominated by the emission of the spine. For comparison, we report historical data for the well-known blazar S5 0716+714 (data from Tavecchio \& Ghisellini 2009 and references therein).

Note that, as observed, the model naturally predicts a very rapid decrease of the emission level above $10 \mathrm{GeV}$, due to the decreasing efficiency of the IC scattering in the Klein-Nishina regime. The position of this break is tightly constrained by the Fermi-LAT spectrum and MAGIC upper limit. In our model, this is critically dependent on the value of the frequency of the target photons for the IC scattering that in the spine-layer scenario are mainly those coming from the spine (and scattered by the electrons in the layer). Therefore, the determination of the cutoff frequency between the Fermi-LAT and the MAGIC band allows us to infer the peak frequency of the synchrotron component of the spine. For instance, assuming that the peak of the spine is at IR frequencies or below (using for the layer the same parameters adopted above), we predict a flux in the MAGIC band above the measured upper limit. This argument allows us to fix the synchrotron peak of the spine at optical-UV frequencies. This, in turn, assures that the beamed counterpart of NGC 1275 is an intermediate BL Lac object, as the chosen S5 0716+714. In conclusion, the knowledge of the upper limit at the low-energy end of the MAGIC band offers us the important possibility of having independent limits on the characteristics of the emission of the (otherwise invisible) spine and thus of constraining the kind of beamed counterpart of this radio galaxy. Future observations can confirm or rule out our interpretation. In particular, the detection of photons above $\sim 100 \mathrm{GeV}$ would be challenging for the scenario depicted here, requiring major changes in the emission properties of the spine.

\section{CONCLUSIONS}

The Perseus cluster was observed by MAGIC during 2008 November and December resulting in $24.4 \mathrm{hr}$ effective observation time of very high data quality. No significant excess of $\gamma$-ray was detected beyond the energy threshold of $80 \mathrm{GeV}$.

Using simplified assumptions (power-law CR spectra, constant ratio of CR-to-thermal energy density) that have been adopted in earlier work, we obtain a limit on the CR energy of $E_{\mathrm{CR}} / E_{\mathrm{th}}<5 \%$. This limit could be tightened furthermore by considering an adiabatically contracted $\mathrm{CR}$ population during the formation of the cooling flow yielding $E_{\mathrm{CR}} / E_{\mathrm{th}}<3 \%$. This would be the most stringent constraint on the CR energy using $\gamma$-ray observations to date. Using cosmological cluster simulations, it turns out that these assumptions are not fulfilled for CR populations that have been accelerated by structure formation shocks: while the adiabatic model seems to match the simulated $\mathrm{CR}$ profiles toward the center very well, the expected ratio of CR-to-thermal pressure increases toward the peripheral cluster regions causing the volume-averaged pressure across the entire cluster to increase by a factor of 2 . In addition, the CR spectral distribution shows a concave curvature with a spectrum that flattens toward high energies with a spectral index of $\Gamma \simeq-2.2$ in the $\mathrm{TeV}$ regime. This implies that the CR pressure is enhanced by an additional factor of almost 2 . Using our simulated flux, we obtained an upper limit on the CR-to-thermal pressure averaged across the entire cluster volume of $\left\langle X_{\mathrm{CR}}\right\rangle<8 \%$ and $<4 \%$ for the cluster core region. This corresponds to an upper limit on the CR energy of $E_{\mathrm{CR}} / E_{\mathrm{th}}<13 \%$ and $<6.5 \%$, respectively. We note that this is the first work where results from cosmological simulations and observational data analysis are combined. This demonstrates the need for cosmological simulations in order to more reliably predict CR spectra which provide a safeguard against too simplified and optimistic models which then lead to limits that are too tight.

The upper limits resulting from the data analysis are a factor of $\simeq 2$ larger than our conservative model prediction for the CR-induced $\gamma$-ray emission and hence in agreement with our cosmological cluster simulations. Future, more sensitive measurements will be able to put interesting constraints on the maximum shock acceleration efficiency. Using minimum $\gamma$-ray flux arguments, we show that improving the sensitivity of this observation by a factor of about 7 will enable us to finally critically test the hadronic model for the Perseus radio minihalo: a non-detection of $\gamma$-ray emission at this level implies CR fluxes that are too small to produce enough electrons through hadronic interactions with the ambient gas protons to explain the observed synchrotron emission.

As DM dominates the cluster mass, significant $\gamma$-ray emission resulting from its annihilation is also expected. With the assumed particle physics model, one of the most optimistic allowed scenarios (Sánchez-Conde et al. 2007) with the neutralino as a DM particle, the boost factor for the typically expected DM annihilation-induced emission is constrained to $<10^{4}$. Note that for this estimation, we neglected possible contributions from internal bremsstrahlung or Sommerfeld enhancement as well as enhancement factors due to substructures.

The upper limits obtained for the NGC 1275 emission are consistent with the recent detection by the Fermi-LAT satellite. In this case a one-zone SSC model for the entire emission is implausible, since the large separation in frequency between the two peaks would require extremely large values of the Doppler factor for the jet (Tavecchio \& Ghisellini 2008). The most direct way to overcome this problem is to assume two emission regions, as in the spine-layer model (Ghisellini et al. 2005) which explains the radio galaxy emission. 
While no galaxy cluster has been detected in $\gamma$-rays up to now, our estimations indicate that Perseus is among the most promising clusters to be detected by IACTs. Using the newly inaugurated MAGIC second telescope and operating the telescopes in a stereo mode (Colin et al. 2009), a total observation time of about $150 \mathrm{hr}$ may give us a chance to detect the CR-induced $\gamma$-ray emission or to definitively probe the validity of the hadronic model of radio (mini-)halos. As the emission of NGC 1275 dominates the accessible energy range of the Fermi-LAT satellite, it could potentially hinder the satellite from detecting the CR as well as the DM-induced $\gamma$-ray emission in this cluster. Similar problems might arise in other clusters. Therefore, the IACTs will play a crucial role in the quest for $\gamma$-ray emission from galaxy clusters.

We thank the anonymous referee for valuable comments. We thank Volker Springel for the important work on CR implementation into the GADGET code and comments on the paper. We also thank Yoel Rephaeli for the useful comments on the paper and Eugene Churazov for providing us XMM-Newton $\mathrm{X}$-ray contours. We thank the Instituto de Astrofisica de Canarias for the excellent working conditions at the Observatorio del Roque de los Muchachos in La Palma. The support of the German BMBF and MPG, the Italian INFN, and Spanish MICINN is gratefully acknowledged. This work was also supported by ETH Research Grant TH 34/043, by the Polish MNiSzW Grant N N203 390834, by the YIP of the Helmholtz Gemeinschaft, and by grant DO02-353 of the Bulgarian National Science Fund.

\section{REFERENCES}

Abdo, A. A., et al. 2009, ApJ, 699, 31

Acciari, V. A., et al. 2009, arXiv:0911.0740

Aharonian, F., et al. 2006, Science, 314, 1424

Aharonian, F., et al. 2009a, A\&A, 495, 27

Aharonian, F., et al. 2009b, A\&A, 502, 437

Aharonian, F., et al. 2009c, ApJ, 695, L40

Ajello, M., et al. 2009, ApJ, 690, 367

Albert, J., et al. 2008a, Nucl. Instrum. Methods Phys. Res. A, 594, 407

Albert, J., et al. 2008b, Nucl. Instrum. Methods Phys. Res. A, 588, 424

Albert, J., et al. 2008c, ApJ, 674, 1037

Albert, J., et al. 2008d, ApJ, 679, 428

Aliu, E., et al. 2009a, ApJ, 697, 1299

Aliu, E., et al. 2009b, Astropart. Phys., 30, 293

Angel, J. R. P., \& Stockman, H. S. 1980, ARA\&A, 18, 321

Balmaverde, B., Capetti, A., \& Grandi, P. 2006, A\&A, 451, 35

Begelman, M. C., Fabian, A. C., \& Rees, M. J. 2008, MNRAS, 384, L19

Berezinsky, V. S., Blasi, P., \& Ptuskin, V. S. 1997, ApJ, 487, 529

Bergström, L., \& Hooper, D. 2006, Phys. Rev. D, 73, 063510

Blasi, P., \& Colafrancesco, S. 1999, Astropart. Phys., 12, 169

Blasi, P., Gabici, S., \& Brunetti, G. 2007, Int. J. Mod. Phys. A, 22, 681

Breiman, L. 2001, Mach. Learn., 45, 5

Bringmann, T., Bergström, L., \& Edsjö, J. 2008, J. High Energy Phys., JHEP01(2008)049

Brunetti, G., Blasi, P., Cassano, R., \& Gabici, S. 2004, MNRAS, 350, 1174

Brunetti, G., \& Lazarian, A. 2007, MNRAS, 378, 245

Brunetti, G., Setti, G., Feretti, L., \& Giovannini, G. 2001, MNRAS, 320, 365

Brunetti, G., Venturi, T., Dallacasa, D., Cassano, R., Dolag, K., Giacintucci, S., \& Setti, G. 2007, ApJ, 670, L5

Bullock, J. S., Kolatt, T. S., Sigad, Y., Somerville, R. S., Kravtsov, A. V., Klypin,

A. A., Primack, J. R., \& Dekel, A. 2001, MNRAS, 321, 559

Carilli, C. L., \& Taylor, G. B. 2002, ARA\&A, 40, 319

Celotti, A., \& Ghisellini, G. 2008, MNRAS, 385, 283

Chiaberge, M., Capetti, A., \& Celotti, A. 1999, A\&A, 349, 77

Churazov, E., Forman, W., Jones, C., \& Böhringer, H. 2003, ApJ, 590, 225

Clarke, T. E. 2004, J. Korean Astron. Soc., 37, 337

Clarke, T. E., Kronberg, P. P., \& Böhringer, H. 2001, ApJ, 547, L111

Colin, P., et al. 2009, arXiv:0907.0960

Dennison, B. 1980, ApJ, 239, L93
Dolag, K., Bykov, A. M., \& Diaferio, A. 2008, Space Sci. Rev., 134, 311

Dolag, K., \& Enßlin, T. A. 2000, A\&A, 362, 151

Domainko, W., Nedbal, D., Hinton, J. A., \& Martineau-Huynh, O. 2009, Int. J. Mod. Phys. D, 18, 1627

Domingo-Santamaria, E., Flix, J., Rico, J., Scalzotto, V., \& Wittek, W. 2005, Proc. 29th Int. Cosmic Ray Conf., ed. B. Sripathi Acharya et al. (Mumbai: Tata Institute of Fundamental Research) 5, 363

Dunn, R. J. H., Fabian, A. C., \& Sanders, J. S. 2006, MNRAS, 366, 758

Edge, A. C., Stewart, G. C., \& Fabian, A. C. 1992, MNRAS, 258, 177

Enßlin, T. A., Biermann, P. L., Kronberg, P. P., \& Wu, X.-P. 1997, ApJ, 477, 560

Enßlin, T. A., Pfrommer, C., Springel, V., \& Jubelgas, M. 2007, A\&A, 473, 41 Enßlin, T. A., \& Vogt, C. 2006, A\&A, 453, 447

Enßlin, T. A., Wang, Y., Nath, B. B., \& Biermann, P. L. 1998, A\&A, 333, L47

Evans, N. W., Ferrer, F., \& Sarkar, S. 2004, Phys. Rev. D, 69, 123501

Fabian, A. C., Sanders, J. S., Taylor, G. B., Allen, S. W., Crawford, C. S., Johnstone, R. M., \& Iwasawa, K. 2006, MNRAS, 366, 417

Falceta-Goncalves, D., de Gouveia Dal Pino, E. M., Gallagher, J. S., \& Lazarian, A. 2010, ApJ, 708, L57

Feretti, L. 2003, in ASP Conf. Ser. 301, Matter and Energy in Clusters of Galaxies, ed. S. Bowyer \& C.-Y. Hwang (San Francisco, CA: ASP) 143

Ferrari, C., Govoni, F., Schindler, S., Bykov, A. M., \& Rephaeli, Y. 2008, Space Sci. Rev., 134, 93

Fomin, V. P., Stepanian, A. A., Lamb, R. C., Lewis, D. A., Punch, M., \& Weekes, T. C. 1994, Astropart. Phys., 2, 137

Forman, W., et al. 2003, arXiv:astro-ph/0301476

Fujita, Y., Kohri, K., Yamazaki, R., \& Kino, M. 2007, ApJ, 663, L61

Galante, N., et al. 2009, arXiv:0907.5000

Geller, M. J., Bruno, M. S., \& Turner, E. L. 1979, ApJ, 230, L141

Ghisellini, G., \& Tavecchio, F. 2008, MNRAS, 386, L28

Ghisellini, G., Tavecchio, F., \& Chiaberge, M. 2005, A\&A, 432, 401

Gitti, M., Brunetti, G., \& Setti, G. 2002, A\&A, 386, 456

Hardcastle, M. J., Cheung, C. C., Feain, I. J., \& Stawarz, Ł. 2009, MNRAS, 393, 1041

Heinz, S., Reynolds, C. S., \& Begelman, M. C. 1998, ApJ, 501, 126

Helder, E. A., et al. 2009, Science, 325, 719

Hernquist, L., \& Katz, N. 1989, ApJS, 70, 419

Hillas, A. M. 1985, in Proc. 19th Int. Cosmic Ray Conf., ed. F. C. Jones (Washington, DC: NASA) 3, 445

Inoue, S., Aharonian, F. A., \& Sugiyama, N. 2005, ApJ, 628, L9

Inoue, S., \& Sasaki, S. 2001, ApJ, 562, 618

Jaffe, W. J. 1977, ApJ, 212, 1

Jeltema, T. E., Kehayias, J., \& Profumo, S. 2009, Phys. Rev. D, 80, 023005

Jubelgas, M., Springel, V., Enßlin, T., \& Pfrommer, C. 2008, A\&A, 481, 33

Kang, H., \& Jones, T. W. 2005, ApJ, 620, 44

Katz, N., \& White, S. D. M. 1993, ApJ, 412, 455

Kellermann, K. I., et al. 2004, ApJ, 609, 539

Kim, K.-T., Kronberg, P. P., \& Tribble, P. C. 1991, ApJ, 379, 80

Kiuchi, R., et al. 2009, ApJ, 704, 240

Kochanek, C. S., White, M., Huchra, J., Macri, L., Jarrett, T. H., Schneider, S. E., \& Mader, J. 2003, ApJ, 585, 161

Kravtsov, A. V., Klypin, A. A., Bullock, J. S., \& Primack, J. R. 1998, ApJ, 502, 48

Kuhlen, M., Diemand, J., \& Madau, P. 2008, ApJ, 686, 262

Lattanzi, M., \& Silk, J. 2009, Phys. Rev. D, 79, 083523

Li, T.-P., \& Ma, Y.-Q. 1983, ApJ, 272, 317

Loeb, A., \& Waxman, E. 2000, Nature, 405, 156

McNamara, B. R., \& Nulsen, P. E. J. 2007, ARA\&A, 45, 117

Miniati, F. 2002, MNRAS, 337, 199

Miniati, F. 2003, MNRAS, 342, 1009

Miniati, F., Jones, T. W., Kang, H., \& Ryu, D. 2001a, ApJ, 562, 233

Miniati, F., Ryu, D., Kang, H., \& Jones, T. W. 2001b, ApJ, 559, 59

Navarro, J. F., Frenk, C. S., \& White, S. D. M. 1997, ApJ, 490, 493

Ohno, H., Takizawa, M., \& Shibata, S. 2002, ApJ, 577, 658

Otte, N., et al. 2009, arXiv:0907.4826

Pedlar, A., Ghataure, H. S., Davies, R. D., Harrison, B. A., Perley, R., Crane, P. C., \& Unger, S. W. 1990, MNRAS, 246, 477

Peebles, P. J. E. 1993, in Principles of Physical Cosmology, ed. P. J. E. Peebles (Princeton, NJ: Princeton Univ. Press)

Perkins, J. S. 2008, in AIP Conf. Ser. 1085, High Energy Gamma-ray Astronomy, ed. F. A. Aharonian, W. Hofmann, \& F. Rieger (Melville, NY: AIP), 569

Perkins, J. S., et al. 2006, ApJ, 644, 148

Petrosian, V., Bykov, A., \& Rephaeli, Y. 2008, Space Sci. Rev., 134, 191

Pfrommer, C. 2008, MNRAS, 385, 1242

Pfrommer, C., \& Enßlin, T. A. 2003, A\&A, 407, L73

Pfrommer, C., \& Enßlin, T. A. 2004a, A\&A, 413, 17 
Pfrommer, C., \& Enßlin, T. A. 2004b, MNRAS, 352, 76

Pfrommer, C., Enßlin, T. A., \& Springel, V. 2008, MNRAS, 385, 1211

Pfrommer, C., Enßlin, T. A., Springel, V., Jubelgas, M., \& Dolag, K. 2007, MNRAS, 378, 385

Pfrommer, C., Springel, V., Enßlin, T. A., \& Jubelgas, M. 2006, MNRAS, 367, 113

Pinzke, A., Pfrommer, C., \& Bergström, L. 2009, Phys. Rev. Lett., 103, 181302

Reimer, O., Pohl, M., Sreekumar, P., \& Mattox, J. R. 2003, ApJ, 588, 155

Reiprich, T. H., \& Böhringer, H. 2002, ApJ, 567, 716

Rephaeli, Y., Nevalainen, J., Ohashi, T., \& Bykov, A. M. 2008, Space Sci. Rev., 134,71

Rolke, W. A., López, A. M., \& Conrad, J. 2005, Nucl. Instrum. Methods Phys. Res. A, 551, 493

Rosati, P., Borgani, S., \& Norman, C. 2002, ARA\&A, 40, 539

Sánchez-Conde, M. A., Prada, F., Łokas, E. L., Gómez, M. E., Wojtak, R., \& Moles, M. 2007, Phys. Rev. D, 76, 123509

Sarazin, C. L. 1988, in Cambridge Astrophys. Ser., X-ray Emission from Clusters of Galaxies, ed. C. L. Sarazin (Cambridge: Cambridge Univ. Press) Sarazin, C. L. 2002, in ASSL 272, Merging Processes in Galaxy Clusters, ed. L. Feretti, I. M. Gioia, \& G. Giovannini (Dordrecht: Kluwer), 1

Schlickeiser, R., Sievers, A., \& Thiemann, H. 1987, A\&A, 182, 21

Schuecker, P., Finoguenov, A., Miniati, F., Böhringer, H., \& Briel, U. G. 2004, A\&A, 426, 387
Springel, V. 2005, MNRAS, 364, 1105

Springel, V., \& Hernquist, L. 2002, MNRAS, 333, 649

Springel, V., Yoshida, N., \& White, S. D. M. 2001, New Astron., 6, 79

Springel, V., et al. 2008a, MNRAS, 391, 1685

Springel, V., et al. 2008b, Nature, 456, 73

Strigari, L. E., Koushiappas, S. M., Bullock, J. S., \& Kaplinghat, M. 2007, Phys. Rev. D, 75, 083526

Tavecchio, F., \& Ghisellini, G. 2008, MNRAS, 385, L98

Tavecchio, F., \& Ghisellini, G. 2009, MNRAS, 394, L131

Taylor, G. B., Fabian, A. C., \& Allen, S. W. 2002, MNRAS, 334, 769

Taylor, G. B., Gugliucci, N. E., Fabian, A. C., Sanders, J. S., Gentile, G., \& Allen, S. W. 2006, MNRAS, 368, 1500

Taylor, G. B., Vermeulen, R. C., Readhead, A. C. S., Pearson, T. J., Henstock, D. R., \& Wilkinson, P. N. 1996, ApJS, 107, 37

Totani, T., \& Kitayama, T. 2000, ApJ, 545, 572

Urry, C. M., \& Padovani, P. 1995, PASP, 107, 803

Veilleux, S., Cecil, G., \& Bland-Hawthorn, J. 2005, ARA\&A, 43, 769

Vestrand, W. T. 1982, AJ, 87, 1266

Vogt, C., \& Enßlin, T. A. 2005, A\&A, 434, 67

Voit, G. M. 2005, Rev. Mod. Phys., 77, 207

Völk, H. J., Aharonian, F. A., \& Breitschwerdt, D. 1996, Space Sci. Rev., 75 279

Yoshida, N., Sheth, R. K., \& Diaferio, A. 2001, MNRAS, 328, 669 